\title{
Fine-Grained Topography and Modularity of the Macaque Frontal Pole Cortex Revealed by Anatomical Connectivity Profiles
}

\author{
Bin $\mathrm{He}^{1,2,3} \cdot$ Long $\mathrm{Cao}^{2,5} \cdot$ Xiaoluan $\mathrm{Xia}^{2,8} \cdot$ Baogui Zhang ${ }^{2,3} \cdot$ Dan Zhang ${ }^{10}$. \\ Bo You ${ }^{1} \cdot$ Lingzhong Fan $^{2,3,4,7} \cdot$ Tianzi Jiang ${ }^{2,3,4,5,6,7,9}$
}

Received: 31 March 2020/Accepted: 30 July 2020/Published online: 27 October 2020

(C) The Author(s) 2020

\begin{abstract}
The frontal pole cortex (FPC) plays key roles in various higher-order functions and is highly developed in non-human primates. An essential missing piece of information is the detailed anatomical connections for finer parcellation of the macaque FPC than provided by the previous tracer results. This is important for understanding the functional architecture of the cerebral cortex. Here, combining cross-validation and principal component analysis, we formed a tractography-based parcellation scheme that applied a machine learning algorithm to divide the macaque FPC ( 2 males and 6 females) into eight subareas using high-resolution diffusion magnetic resonance imaging with the $9.4 \mathrm{~T}$ Bruker system, and then
\end{abstract}

Electronic supplementary material The online version of this article (https://doi.org/10.1007/s12264-020-00589-1) contains supplementary material, which is available to authorized users.

\section{Bo You \\ youbo@hrbust.edu.cn \\ $\bowtie$ Lingzhong Fan \\ lingzhong.fan@ia.ac.cn \\ $\bowtie$ Tianzi Jiang \\ jiangtz@nlpr.ia.ac.cn}

1 School of Mechanical and Power Engineering, Harbin University of Science and Technology, Harbin 150080, China

2 Brainnetome Center, Institute of Automation, Chinese Academy of Sciences, Beijing 100190, China

3 National Laboratory of Pattern Recognition, Institute of Automation, Chinese Academy of Sciences (CAS), Beijing 100190, China

4 Center for Excellence in Brain Science and Intelligence Technology, Institute of Automation, CAS, Beijing 100190, China revealed their subregional connections. Furthermore, we applied improved hierarchical clustering to the obtained parcels to probe the modular structure of the subregions, and found that the dorsolateral FPC, which contains an extension to the medial FPC, was mainly connected to regions of the default-mode network. The ventral FPC was mainly involved in the social-interaction network and the dorsal FPC in the metacognitive network. These results enhance our understanding of the anatomy and circuitry of the macaque brain, and contribute to FPC-related clinical research.

Keywords Macaque . Frontal pole cortex $\cdot$ Anatomical connectivity profile $\cdot$ Parcellation - Neuroimaging · Principal component analysis

5 Key Laboratory for NeuroInformation of the Ministry of Education, School of Life Science and Technology, University of Electronic Science and Technology of China, Chengdu 610054, China

6 The Queensland Brain Institute, University of Queensland, Brisbane, QLD 4072, Australia

7 University of CAS, Beijing 100049, China

8 College of Information and Computer, Taiyuan University of Technology, Taiyuan 030600, China

9 Chinese Institute for Brain Research, Beijing 102206, China

10 Core Facility, Center of Biomedical Analysis, Tsinghua University, Beijing 100084, China 


\section{Abbreviations}

\section{Abbreviations of the Brain Regions}

\section{0}

11

13

23

25

30

31

32

35

44

10471

15584

$10 \mathrm{D}$

$10 \mathrm{M}$

$10 \mathrm{~V}$

$11 \mathrm{~L}$

$11 \mathrm{~m}$

$13 \mathrm{a}$

$13 \mathrm{~L}$

$13 \mathrm{M}$

$14 \mathrm{M}$

$14 \mathrm{o}$

$23 \mathrm{a}$

$23 \mathrm{~b}$

$23 \mathrm{c}$

$24 / 23 \mathrm{a}$

$24 / 23 b$

$24 \mathrm{a}$

$24 \mathrm{~b}$

$24 \mathrm{c}$

$29 \mathrm{a}$

$45 \mathrm{~A}$

$45 \mathrm{~B}$

46D

$46 \mathrm{~V}$

47(12)

47(12)L

47(12)O

6VR(F5)

$8 \mathrm{AD}$

$8 \mathrm{AV}$

9/46D

9/46V

9L

$9 \mathrm{M}$

AA

Acb

AI

AO
Area 10 of cortex

Area 11 of cortex

Area 13 of cortex

Area 23 of cortex

Area 25 of cortex

Area 30 of cortex

Area 31 of cortex

Area 32 of cortex

Area 35 of cortex

Area 44 of cortex

Area 9/32 of cortex

Area 9/46 of cortex

Area 10 of cortex, dorsal part

Area 10 of cortex, medial part

Area 10 of cortex, ventral part

Area 11 of cortex, lateral part

Area 11 of cortex, medial part

Area 13a of cortex

Area 13 of cortex, lateral part

Area 13 of cortex, medial part

Area 14 of cortex, medial part

Area 14o

Area 23a of cortex

Area 23b of cortex

Area 23c of cortex

Area 24/23a of cortex

Area $24 / 23 b$ of cortex

Area 24a of cortex

Area $24 b$ of cortex

Area $24 c$ of cortex

Area 29a of cortex

Area 45A of cortex

Area 45B of cortex

Area 46D of cortex

Area $46 \mathrm{~V}$ of cortex

Area 47 (old 12) of cortex

Area 47 (old 12) of cortex, lateral part

Area 47 (old 12) of cortex, orbital part

Area 6 of cortex, rostral ventral part

Area 8 of cortex, anterodorsal part

Area 8 of cortex, anteroventral part

Area 9/46 of cortex, dorsal part

Area 9/46 of cortex, ventral part

Area 9 of cortex, lateral part

Area 9 of cortex, medial part

Anterior amygdaloid area

Accumbens nucleus

Agranular insular cortex

Anterior olfactory nucleus
Apul Anterior pulvinar

Atha Anterior thalamic nucleus

BL\#2 Basolateral amygdaloid nucleus

BLD Basolateral amygdaloid nucleus, dorsal part

BM\#3 Basomedial amygdaloid nucleus

BM\#4 Basal nucleus (Meynert)

BST Bed nucleus of the stria terminalis central division

BSTIA Bed nucleus of the stria terminalis intraamygdaloid division

$\mathrm{Cd} \quad$ Caudate nucleus

$\mathrm{Ce} \quad$ Central amygdaloid nucleus

$\mathrm{Cl}$ 2 Centrolateral thalamic nucleus

$\mathrm{CM \# 2}$ Central medial thalamic nucleus

CMnM Centromedial thalamic nucleus, medial part

Den Dorsal endopiriform nucleus

DI Dysgranular insular cortex

GP Globus pallidus

$\mathrm{Gu} \quad$ Gustatory cortex

HDB Nucleus of the horizontal limb of the diagonal band

Hy Hypothalamus

IAM Interanteromedial thalamic nucleus

IMD Intermediodorsal thalamic nucleus

IPro Insular proisocortex

Ipul Inferior pulvinar

La\#3 Lateral amygdaloid nucleus

Ldsf Lateral dorsal thalamic nucleus, superficial part

Lpul Lateral pulvinar

LV Lateral ventricles

MB Midbrain

MD Mediodorsal thalamic nucleus

$\mathrm{Me} \quad$ Medial amygdaloid nucleus

MPul Medial pulvinar

OPAl Orbital periallocortex

OPro Orbital proisocortex

PaIL Parainsular cortex

PaS Parasubiculum

Pf\#2 Parafascicular thalamic nucleus

PGM Area PGM/31 of cortex

Pir Piriform cortex

ProKM Prokoniocortex, medial part

ProM Area ProM (promotor)

ProST Prostriate area

PrS Presubiculum

$\mathrm{Pu} \quad$ Putamen

Pul\#1 Pulvinar nuclei

Pvt Paraventricular thalamus

R\#4 Reticular thalamic nucleus

R36 The perirhinal cortex

$\mathrm{Re} \quad$ Reuniens thalamic nucleus

Se Septum

SI Substantia innominata 


$\begin{array}{ll}\text { ST1 } & \text { Superior temporal sulcus area 1 } \\ \text { ST2 } & \text { Superior temporal sulcus area } \\ \text { ST3 } & \text { Superior temporal sulcus area 3 } \\ \text { TAa } & \text { Temporal area TAa } \\ \text { TPPro } & \text { Temporopolar proisocortex } \\ \text { TTPAl } & \text { Temporopolar periallocortex } \\ \text { Tu } & \text { Olfactory tubercle } \\ \text { VA } & \text { Ventral anterior thalamic nucleus } \\ \text { VL } & \text { Ventral lateral thalamic nucleus } \\ \text { VP\#3 } & \text { Ventral pallidum }\end{array}$

\section{Other Abbreviations}

$\begin{array}{ll}\text { ANTs } & \text { Advanced normalization tools } \\ \text { aspd } & \text { Anterior supraprincipal dimple } \\ \text { cgs } & \text { Cingulate sulcus } \\ \text { DMN } & \text { Default-mode network } \\ \text { dMRI } & \text { Diffusion magnetic resonance imaging } \\ \text { DTI } & \text { Diffusion tensor imaging } \\ \text { ESIN } & \text { Exclusively social interaction network } \\ \text { fMRI } & \text { Functional magnetic resonance imaging } \\ \text { MIPAV } & \text { Medical image processing, analysis, and visu- } \\ & \text { alization software } \\ \text { morbs } & \text { Medial orbital sulcus } \\ \text { MRI } & \text { Magnetic resonance imaging } \\ \text { PCA } & \text { Principal component analysis } \\ \text { ps } & \text { Principal sulcus } \\ \text { pspd } & \text { Posterior supraprincipal dimple } \\ \text { ROI } & \text { Region of interest } \\ \text { ros } & \text { Rostral sulcus } \\ \text { SIN } & \text { Social-interaction network }\end{array}$

\section{Introduction}

The macaque frontal pole cortex (FPC) has a homotypical cytoarchitecture and a location relative to other prefrontal regions that is similar to that of humans [1], which means that it has the potential to be an excellent model for understanding the mechanisms of the human brain $[2,3]$. As a portion of the prefrontal cortex, this area that has undergone more extensive evolution [4], and it is a latedeveloping area of the neocortex [5]. The FPC has a singularly high neuronal density and rich dendritic spines, which together suggest complex functions and multiple areas of cytoarchitectonic differentiation. In addition, the functional complexity of the FPC varies between species, which makes it a focal point for comparisons across species. Especially, as the core area involved in decisionmaking in the executive system [6,7], the FPC has been pinpointed as a unique area that could separate humans from other primates with respect to higher cognitive powers $[8,9]$.
Although a variety of findings suggest that the macaque FPC can be divided into multiple functional subareas with different connectivity $[10,11]$, this area still lacks specialized research on its anatomical connections and a detailed parcellation map. Much of the previous work on this region primarily focused on cytoarchitecture and tract-tracing techniques [12-15]. The early researchers defined this area as Brodmann area (BA) 10 in humans and BA 12 in nonhuman primates [16]. Subsequently, this area was reclassified as BA 10 in non-human primates by Walker [17]. Recently, three primary studies have revealed markedly different cytoarchitectonic parcellation results [18-20], and many trace-injection experiments involving the FPC were based on previous rough maps of this area. In addition, the FPC has been suggested to be potentially associated with the default-mode network (DMN) [21, 22]. Statistical maps of enhanced activation have revealed that the FPC is involved in the social-interaction network (SIN) [23]. In macaque monkeys, Miyamoto, Setsuie, Osada, Miyashita [24] found that the FPC is recruited for the metacognitive judgment of non-experienced events by fMRI experiments. The inactivation of this area does not affect the detection of non-experienced events, but selectively impairs the metacognition of non-experienced events. From a different perspective, studies based on the diffusion tensor imaging (DTI) connectivity could further improve our understanding of the relationship between the macaque FPC and different functional networks, including the DMN, SIN, and metacognitive networks, but relevant studies are lacking. The above issues, which are both important and controversial, hint at the urgency of obtaining a detailed understanding of this region; however, to our knowledge, the macaque FPC remains one of the least understood brain areas [25]. Moreover, the traditional parcellation method based on cytoarchitectonics is not only limited to noninvasive approaches, but is also limited by the number of samples and lack of consideration of individual variation. Many trace-injection studies related to the FPC have been based on previous rough parcellation maps and relevant studies based on DTI are still a rarity.

In view of the importance of the diversity of functions of the monkey FPC and the lack of detailed anatomical connection information in comparison with the previous tracer results [19, 26, 27], as well as to pave the way for a systematic follow-up study using tracer injections, a study of the topological organization properties of the macaque FPC is necessary and attractive. Recently, connectivitybased parcellation (CBP) has been a powerful framework for mapping the human brain [28-30] and may provide a better picture of regional parcellation and anatomical connectivity information $[31,32]$ as well as allowing the target areas of tracer injections to be chosen less blindly. In this study, we provided a tractography-based parcellation 
scheme that applied a machine-learning algorithm to obtain a fine-grained subdivisions of the macaque FPC, and then revealed their subregional connections. Exploring the modular structure of a community and the anatomical connectivity patterns of different functional networks could help understand brain mechanisms and evolution, which contributes to FPC-related clinical research.

\section{Materials and Methods}

To study the topological organization properties of the macaque FPC, three research objectives were established and the corresponding work was carried out. The overall workflow is shown in Fig. 1. First, we used DTI data to divide the macaque FPC into different subregions; then we explored the anatomical connectivity patterns of each subdivision. Furthermore, we proposed an improved hierarchical clustering algorithm to explore the modular structure of the community for the bilateral subregions.

\section{Macaque Brain Specimens}

The rhesus macaques (Macaca mulatta) were obtained from Kunming Institute of Zoology, Chinese Academy of Sciences [33] (details in Table 1). All experimental procedures were conducted according to the policies set forth by the National Institutes of Health Guide for the Care and Use of Laboratory Animals, and approved by the
Animal Care and Use Committee of the Institute of Automation, Chinese Academy of Sciences. They were judged by the veterinarian as appropriate subjects for euthanasia due to serious illnesses (acute gastroenteritis and enteritis). Each animal was intraperitoneally administered an overdose of pentobarbital $[100 \mathrm{mg} / \mathrm{kg}$, Sigma Aldrich (Shanghai) Trading Co., Ltd, Shanghai]. After verifying the status of deep anesthesia, they were transcardially perfused first with Phosphate-buffered saline (PBS) containing 1\% heparin [pH 7.4, Sigma Aldrich (Shanghai) Trading Co., Ltd, Shanghai], followed by pre-cooled PBS containing 4\% paraformaldehyde [Sigma Aldrich (Shanghai) Trading Co., Ltd, Shanghai]. Five minutes after starting the perfusion, the rate was lowered to $1 \mathrm{~mL} / \mathrm{min}$ from an initial rate of $20 \mathrm{~mL} / \mathrm{min}$, and the entire perfusion lasted $2 \mathrm{~h}$. The head was then removed and stored in PBS containing $4 \%$ paraformaldehyde. Then, the skull was carefully removed to expose the whole brain for MRI scanning. No apparent structural anomalies were found in any of the brains used in the present study.

\section{MRI Acquisition}

All the macaque MRI data were obtained using a 9.4T horizontal animal MRI system [Bruker Biospec 94/30 USR, with Paravision 6.0.1 (Ettlingen,Baden-Württemberg, Germany)]. Radiofrequency (RF) transmission and reception were achieved with a 154-mm inner-diameter quadrature RF coil. The SpinEcho DTI sequence used for

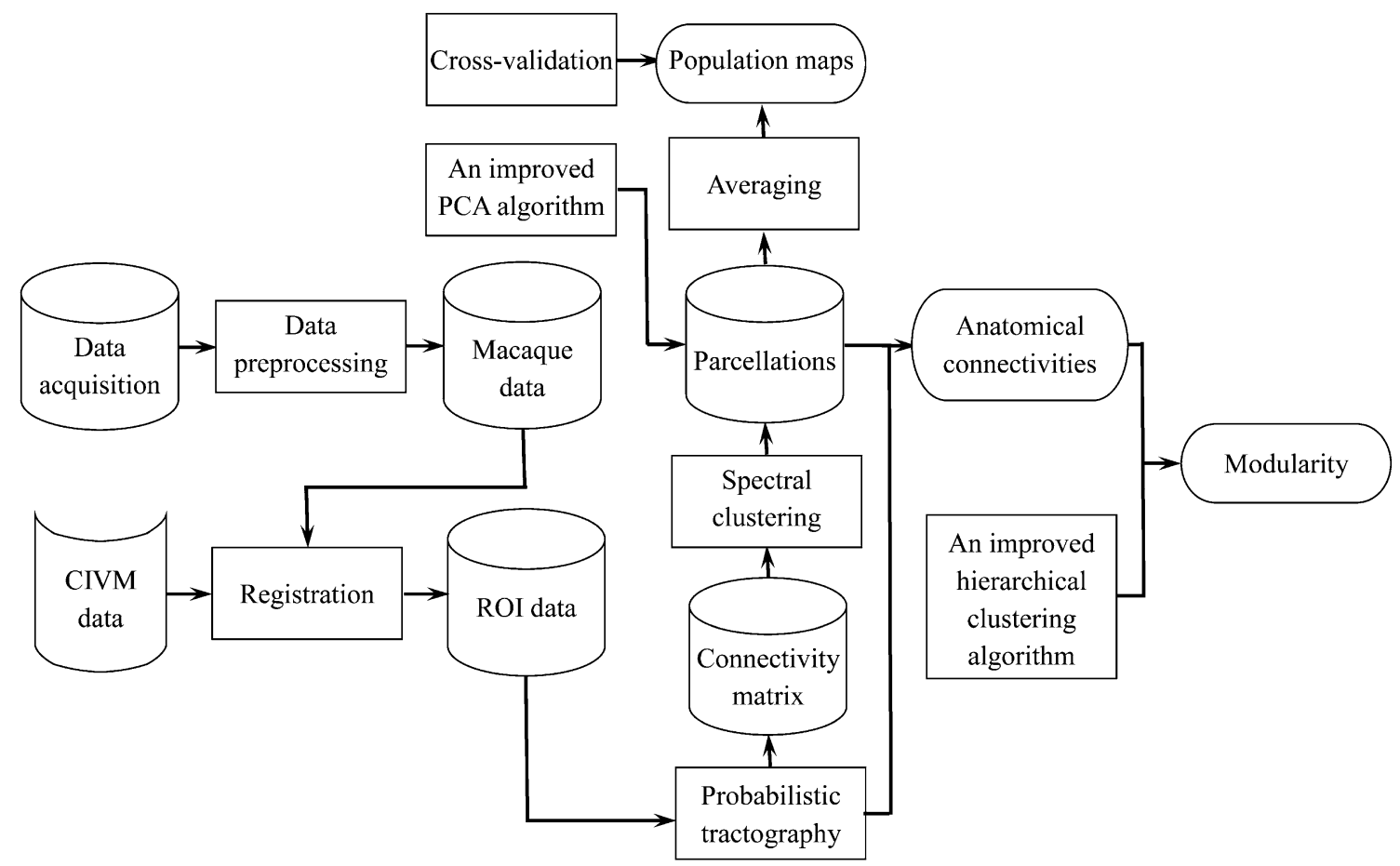

Fig. 1 The overall workflow of this study. 
Table 1 Information about the eight monkey brains.

\begin{tabular}{lllrl}
\hline Perfusion date & Number & Gender & Age & Weight $(\mathrm{kg})$ \\
\hline $2016 / 05 / 09$ & 93310 & Female & 23 & 3.24 \\
& 08046 & Female & 8 & 3.58 \\
& 12027 & Male & 4 & 3.06 \\
$2016 / 05 / 10$ & 12411 & Male & 4 & 2.89 \\
& 01006 & Female & 15 & 3.57 \\
& 04084 & Female & 12 & 4.23 \\
& 10427 & Female & 6 & 3.69 \\
& 11402 & Female & 5 & 2.9
\end{tabular}

the DTI data provided the main parameters: 74 slices, echo time $(\mathrm{TE})=22 \mathrm{~ms}$, repetition time $(\mathrm{TR})=9800 \mathrm{~ms}$, field of view $(\mathrm{FOV})=94 \times 66 \mathrm{~mm}^{2}$, flip angle $(\mathrm{FA})=90^{\circ}$, acquisition matrix $=140 \times 110$, and resolution $=0.6 \times 0.6$ $\times 0.6 \mathrm{~mm}^{3}$ without gap. This sequence produced a complete set of 64 images, including 4 non-diffusionweighted images $\left(b=0 \mathrm{~s} / \mathrm{mm}^{2}\right)$ and 60 images with noncollinear diffusion gradients $\left(b=1000 \mathrm{~s} / \mathrm{mm}^{2}\right)$ and required $\sim 115 \mathrm{~h}$ of scanning time per specimen. T1-weighted data were acquired using a 2D IR-prepared RARE sequence with these main parameters: 74 slices, $\mathrm{TE}=5.8 \mathrm{~ms}, \mathrm{TR}=$ $4019 \mathrm{~ms}$, inversion time $=750 \mathrm{~ms}$, matrix $=280 \times 220$, FA $=90^{\circ}$, resolution $=0.3 \times 0.6 \times 0.3 \mathrm{~mm}^{3}, \mathrm{FOV}=84 \times 66$ $\mathrm{mm}^{2}$, slice thickness $=0.6 \mathrm{~mm}$, and no gap, requiring $\sim 55$ min. T2-weighted data were obtained in a 2D Turbo RARE sequence with these main parameters: 86 slices, $\mathrm{TE}=30.9$ $\mathrm{ms}, \mathrm{TR}=8464 \mathrm{~ms}, \mathrm{FA}=90^{\circ}$, resolution $=0.3 \times 0.6 \times 0.3$ $\mathrm{mm}^{3}$, matrix $=280 \times 220, \mathrm{FOV}=84 \times 66 \mathrm{~mm}^{2}$, slice thickness $=0.6 \mathrm{~mm}$, and no gap, requiring $\sim 15 \mathrm{~min}$.

\section{Definition of Seed and Target Masks of Macaque FPC}

The macaque FPC seed masks were extracted from a publicly-available post-mortem macaque brain atlas (CIVM, https://scalablebrainatlas.incf.org/macaque/CBCe tal15) [34] and all regional names were found in the list of abbreviations. This atlas is largely consistent with that of Paxinos et al. [18] Nissl-based atlas and has become increasingly popular in macaque studies [35-37]. The mask occupied the most rostral portions of the prefrontal cortex; its dorsal extent was bounded posteriorly by the anterior supraprincipal dimple (aspd) and did not cross the posterior supraprincipal dimple (pspd). In addition, on the medial aspect of the hemisphere, the FPC mask posteriorly bordered the cingulate sulcus (cgs) and in the coronal plane, it was ventrally delimited by the anterior termination of the olfactory sulcus. For each subject, the standard seed mask was wrapped back into individual diffusion space using the inverse of the deformations, and each resulting mask was visually inspected for possible errors and necessary modifications using ITK-SNAP (Philadelphia, Pennsylvania) [38]. To calculate the connectivity matrix and obtain the connectivity fingerprints, we extracted cortical regions and subcortical structures in the same hemisphere from the CIVM atlas as target regions [39]. The extraction approach for the target regions was the same as that for the FPC. Subsequently, we transformed them into individual diffusion space.

\section{Diffusion MRI Data Preprocessing}

The diffusion MRI (dMRI) data were preprocessed using the FMRIB Diffusion Toolbox (FSL version 5.0; https://fsl. fmrib.ox.ac.uk/fsl/fslwiki/FSL), the prominent Medical Image Processing, Analysis, and Visualization software (MIPAV, https://mipav.cit.nih.gov/), and Advanced Normalization Tools (ANTs, http://www.picsl.upenn.edu/ ANTS/), which is a state-of-the-art medical image registration toolkit [40]. The main procedure included the following steps. First, for the CIVM template, we transformed the raw format to the available dMRI space with MIPAV, which enables the quantitative analysis and visualization of medical images in different formats. Second, in the diffusion data from each subject, distortions caused by eddy currents were corrected using the FSL tool [41]. Finally, after conversion into the standard available format with MIPAV, the b0 image of the CIVM template space was coregistered to the individual non-diffusion-weighted images $\left(\mathrm{b}=0 \mathrm{~s} / \mathrm{mm}^{2}\right)$ using ANTs. After the registration, an inverse transformation was performed to transform the seed and target masks for each subject's small cortical areas into native dMRI space.

\section{Probabilistic Diffusion Tractography}

After preprocessing, the macaque FPC was chosen as the seed and probabilistic tractography was carried out for the tractography-based parcellation. This process has been described in the toolbox for connectivity-based parcellation of the monkey brain [42] and is similar to that in another study [43]. Voxelwise estimates of the fiber orientation distribution were computed using Bedpostx. We calculated the probability distributions in two fiber directions at each voxel using a multiple fiber extension [44]. Based on the probability distributions, we then estimated the connectivity probability between each voxel in the seed region and every voxel of the whole brain using PROBTRACKX2 (Oxford, Oxfordshire). Probabilistic tractography was applied by sampling 15,000 streamline fibers per voxel and the step size was set to $0.2 \mathrm{~mm}[45,46]$. To exclude implausible pathways, we restricted how sharply pathways 
could turn, and the default threshold was set to 0.2 . To correct the path distribution for the length of the pathways, we introduced a distance correction. The connection probability between each voxel in the seed region and any other voxel in the brain was obtained by computing the number of traces arriving at the target site. To decrease the number of false-positive connections, we thresholded the path distribution estimates for each subject using a connection probability value $P<20 / 15000$ (20 out of 15,000 samples). Information about the connectivity was stored in an M-by- $\mathrm{N}$ matrix, where $\mathrm{M}$ denotes the number of voxels in the seed mask and $\mathrm{N}$ the number of voxels in the native diffusion space. To de-noise the data and increase computational efficiency, the connectivity profiles for each voxel were down-sampled to 2-mm isotropic voxels [47].

\section{Tractography-Based Parcellation of the Macaque FPC}

Based on the connectivity patterns of all the voxels of the FPC, cross-correlation matrices were computed and fed into spectral clustering [28]. The maximum probability map (MPM) was computed by assigning each voxel of the standard space to the subarea in which it was most likely to be located [48]. First, we transformed the parcellation results from individual diffusion space to the CIVM template. Second, the MPM was computed according to the eight subjects' parcellation results in CIVM space.

After parcellation of the FPC using spectral clustering, the next step was to select the number of clusters. To avoid an arbitrary choice of this number, we used two prevailing validation methods, cross-validation indices to obtain a consistent segmentation for all eight subjects at the group level, and principal component analysis (PCA), to determine the optimal number of clusters across the subjects at the individual diffusion level [42].

\section{Cross-Validation Indices}

The cross-validation offered two indices, Cramer's V [49, 50] and topological distance (TpD) [51], for determining the optimal clustering number. Cramer's $\mathrm{V}$, is an indicator of clustering consistency and has values in the interval $[0,1]$, high values indicating good consistency. The TpD index, which quantifies the similarity of the topological arrangement of putative homologous regions in the bilateral hemispheres across all specimens, further determined the cluster number. The TpD score ranges from 0 to 1 ; a score close to 0 suggests that the two hemispheres have similar topology. The clustering number of local extremum points (peaks and valleys) means better consistency than that of adjacent ones, and in general, the local extrema are recommended as a good solution for each presumptive index $[52,53]$.

\section{Principal Component Analysis}

PCA, which requires no artificial hypothesis or prior knowledge, is a popular statistical framework for determining the clustering number $[30,54]$. To ensure that the number of principal components to be chosen retain enough features and effectively represent the data, we proposed three criteria based on the literature [55, 56]. The first was the cumulative contribution, which means that a cumulative proportion of the variation could be explained by the eigenvalue obtained using the connection data. To obtain the cumulative proportion value (cpv), a threshold must be established. Generally, a sensible threshold is very often in the range $70 \%$ to $90 \%$; it can sometimes be higher or lower depending on the practical details of a particular dataset. In our study, we thresholded the cpv at $>80 \%$. Taking into account individual variation, we allowed a lower limit change of no more than $1 \%$. The second criterion was that only factors with eigenvalues $>1$ or next closest to 1 were retained. Specifically, the latter weak criterion (values close to 1) is like a "factorial scree" for atypical individual variation. The third criterion is a scree test [30, 54]. Briefly, for each subject, a 'connectivity' matrix between the various seeds and the whole brain was derived from the data of the probabilistic tractography. This matrix consisted of columns that indicated the FPC subregion of interest and rows that represented the wholebrain regions. To estimate the number of principal components to extract from each subject, a power curve was plotted by fitting the data, the inflexion point was extracted using a homemade routine written in MatLab (Natick, Massachusetts) R2017b, and all subjects were averaged to obtain a mean cluster value for the left and right hemispheres separately. Meanwhile, we set the difference threshold between the inflection point value of each individual and the average value as 0.5 to ensure that the clustering number among individuals was stable.

\section{Anatomical Connectivity Patterns}

To explore the different anatomical connection patterns of the FPC subdivisions, we first drew $10^{5}$ samples from the fiber orientation distribution for each voxel in the subdivisions to calculate the whole-brain probabilistic fiber tracking [44]. To form the seed mask, each subarea was extracted from the probability map of the FPC at $25 \%$ probability. To reduce the false-positive rate and facilitate the qualitative analysis, we thresholded the connectivity probability value at $3.08 \times 10^{-5}$ at the individual level, which means that at least 3.08 of the $10^{5}$ samples produced 
from each seed voxel were connected [48]. Next, the identified fiber tracts were binarized and transformed into CIVM macaque template space. All the binarized results were averaged to obtain population maps with a threshold of 50\% [39], which means that only those voxels that were present in at least 4 of 8 subjects were mapped, and were then transformed into F99 space for display.

Subsequently, to visualize the differences in the anatomical connectivity of each subarea, we further calculated the anatomical connectivity fingerprints between each subarea and each of the target regions in the CIVM atlas. For the eight subjects, these target brain regions, including the cortical areas and subcortical structures of each subject, were extracted from the CIVM atlas in the same hemisphere using the same method as used to extract the FPC and was subsequently transformed into individual dMRI space. Using their fingerprints, we were able to find the different connectivity properties for each subregion. In addition, we performed similarity analysis of the connections for these clusters to estimate the connection similarity between individuals. Briefly, we computed the correlation coefficients for the seed-to-target connections and obtained the $P$ values for the hypothesis that there was no relationship between the observed phenomena. We defined the threshold of statistical significance as statistically highly significant at $P<0.001$.

Furthermore, we also investigated and summarized other tract-tracing studies involving the macaque FPC to compare their consistency by assessing the repeatability of the connected areas that they identified. As a preliminary qualitative comparison, we collected the regions connected to the FPC from the CoCoMac database [57] and compared them with the regions connected to subdivisions of the FPC. The CoCoMac database provides convincing structural connectivity data for the macaque brain and was a remarkable effort by many researchers. Currently, it is the largest macaque connectivity study, with data extracted from $>400$ published tract-tracing studies of the macaque brain. We also made a further comparison with some of the detailed trace-injection experiments.

Moreover, inspired by a human frontal pole study [50], to reveal a clearer picture of different functional networks from the perspective of anatomical connections, we analyzed the connections between the subregions and the regions of different functional networks. Specifically, we combined the regions that were connected to the DMN [21, 22], SIN [23], and metacognition network [24, 58] and estimated the linkages and differences between the subareas. Additional comparisons with other studies and findings are presented in the Discussion.

\section{Mapping the Hierarchical Module Structure for FPC Subregions}

Exploring the modular structure of a community and the connectivity patterns of different functional networks is important for understanding brain mechanisms and evolution. Many neuroscientific studies [59-61] have revealed that the brain networks share important organizational principles in common, such as modularity, and that topological modules often comprise anatomically neighboring cortical areas. In addition, the modules of brain networks contain both unilateral and bilateral areas [62], and a community structure in the brain can be correlated with functionally localized regions, such as visual, auditory, and central modules [63]. Here, we proposed an improved hierarchical clustering algorithm to examine the subregional members of the bilateral FPC and assign them to clusters. The correlation coefficient matrix of the native connectivity data was fed into the algorithm; as the number of clusters increased, those with high connection similarity were given priority and grouped together. Besides, we calculated the cophenetic correlation coefficient to evaluate and select the optimal clustering scheme. The hierarchical clustering method not only uncovered the modular community structure of the bilateral FPC, but also provided some methods that other researchers can use in making within-species comparisons. In particular, this method can be used for comparisons between parcellations with a greater number of subdivisions and those with fewer subdivisions that have been obtained from studies that use different methods. This approach can also be used to make heuristic comparisons between species, including comparisons of parcellation patterns and connectivity patterns involving different functional networks. Compared with previous studies [33, 43], the advantage of our method is that it is able to automatically select the optimal clustering by introducing the cophenetic correlation coefficient to compare the results of clustering the same data set using different distance calculation methods and clustering algorithms. The cophenetic correlation coefficient scores range from 0 to 1 . The closer the value is to 1 , the more accurately the clustering solution reflects the data.

\section{Results}

\section{Connectivity-Based Parcellation and Subregional Anatomical Connectivity Patterns of the Macaque FPC}

For the macaque FPC, the cross-validation of the spectral clustering data showed that the 8-cluster solution, as local extremum points of Cramer's V, was optimal for a fine 
parcellation (Fig. 2A). The TpD index also selected the 8-cluster solution as optimal (Fig. 2B). These results of validity indices suggested the consistency of the parcellations across subjects and the similarity of the topological organization distribution of the parcellation results between the bilateral hemispheres at the group level [42]. In addition, at the individual diffusion space level, the index results of PCA also suggested a segregation of the FPC into an average of 8 subdivisions for each of the hemispheres (left hemisphere, 8.31, see Fig. 2E; right hemisphere, 8.27, see Fig. 2F).

The eight distinct subareas consisted of four components in the lateral section with the remaining four components in the medial section. These results were transformed and combined into F99 brain space [64] with Caret software [65] to create population-based parcellations of the FPC, and we further presented the probabilistic map for each subarea that could help to understand the consistency between subjects in the topography of the clusters (left hemisphere see Fig. 3; right hemisphere see Fig. S1). To facilitate understanding of the results, we determined the location of each subregion based on histologically defined cortical areas and a topologic map as well as on its anatomical connection information. In describing the location of these subregions, we refer to them with respect to the cgs, principal sulcus (ps), medial orbital sulcus (morbs), rostral sulcus (ros), aspd, and the adjacent areas. In the sagittal plane, a ventrolateral boundary along the direction of the ps separates $\mathrm{C} 4$ from $\mathrm{C} 6$, and another lateral boundary above the rostral ps distinguishes subareas C6 from C8. The dorsolateral boundary above the aspd separates subareas C8 from C7. In the medial FPC, a boundary along the anterior extension of the ros segregates subareas $\mathrm{C} 3$ from $\mathrm{C} 2$, and another boundary around the rostral cgs distinguishes subareas $\mathrm{C} 1$ from $\mathrm{C} 2$. Above the rostral cgs, a dorsomedial boundary separates subareas C5 from $\mathrm{C} 1$.

Furthermore, the anatomical connectivity patterns of each subregion were obtained from the whole-brain probabilistic tractography in native diffusion space by estimating the fiber orientations for each voxel. To minimize the effects of inter-individual variations, the probabilistic patterns of the fiber tracts were then transformed into CIVM space; then an averaged fiber tract map was calculated for each subdivision and displayed in the F99 surface. The anatomical connectivity fingerprints between the subdivisions and other brain structure areas of the CIVM atlas could identify the connectivity differences for each subarea (see Fig. 4, Fig. 5, S2, and S3 for details).

\section{Cluster Cl}

C1 was located in the medial part of the FPC around the rostral tip of the anterior cingulate cortex (ACC), following a medial-to-lateral direction, gradually along the dorsal surface of area 32, then in front of areas $9 / 32$ and 32 . Following an anterior-to-posterior direction, with the extension of the ros, $\mathrm{C} 1$ extended dorsally to the cgs. Above it was Cluster C5, and its dorsal extent was limited by Cluster $\mathrm{C} 8$. Its ventral border was delimited by Cluster $\mathrm{C} 2$ just above the ros, while ventrally it was anteriorly delimited by Cluster C6. This subdivision also encompassed part of the anterior bank of the cgs. Tractography samples seeded from Cluster $\mathrm{C} 1$ to the cortex were mostly distributed in the medial frontal cortex, the adjacent ACC, and the posterior cingulate cortex, including areas 32, 24a, 24b, 9/32, 24/23a, 29, and 10M. At a longer distance, it connected with areas 31, 23, PGM, ProST, TTPAl, and TPPro. In the subcortical results, area $\mathrm{C} 1$ showed a stronger connection with $\mathrm{Re}, \mathrm{IMD}, \mathrm{Cl} 2$, and $\mathrm{Se}$.

\section{Cluster C2}

C2 was located in the medial part of the FPC just below Cluster C1. It lay anterior to area 32, followed a medial-tolateral direction immediately in front of the rostral cgs and also encompassed part of the rostral bank of the cgs. In its dorsal rostral part, it extended to the most posterior part of Cluster C6. Its ventral extent was limited by Cluster C3, which was located in the orbital FPC. In the coronal plane, Cluster 2 was above the smooth extension line of the ps. The connectivity of Cluster $\mathrm{C} 2$ was predominantly with the medial frontal cortex and part of the lateral frontal lobe, including areas 24a, 32, the most anterior FPC, 24b, 24/23a, 14, and 10M. In addition, the subcortical structures, including Se, SI, BM\#4, AA, and Re, shared a stronger connectivity seeded from area $\mathrm{C} 2$.

\section{Cluster C3}

C3 covered the medial orbital part of the FPC. Following a medial-to-lateral direction, its dorsal border was delimited by Clusters $\mathrm{C} 2$ and $\mathrm{C} 6$ and gradually continued along the ventral area of Cluster $\mathrm{C} 4$. Along the lateral extended direction, the morbs separated Cluster $\mathrm{C} 3$ from Cluster $\mathrm{C} 4$. C3 was heavily connected with the orbitofrontal cortex, temporal cortex, and LV, including areas 14, OPAl, 13a, and the anterior and ventral parts of area 10. This cluster had strong connections with the orbital periallocortex, the rostral part of area TL (area 36R), and the temporopolar periallocortex. Compared with the connection strength with subcortical structures, Cluster C3 shared stronger connections with SI, HDB, Se, and BST. 

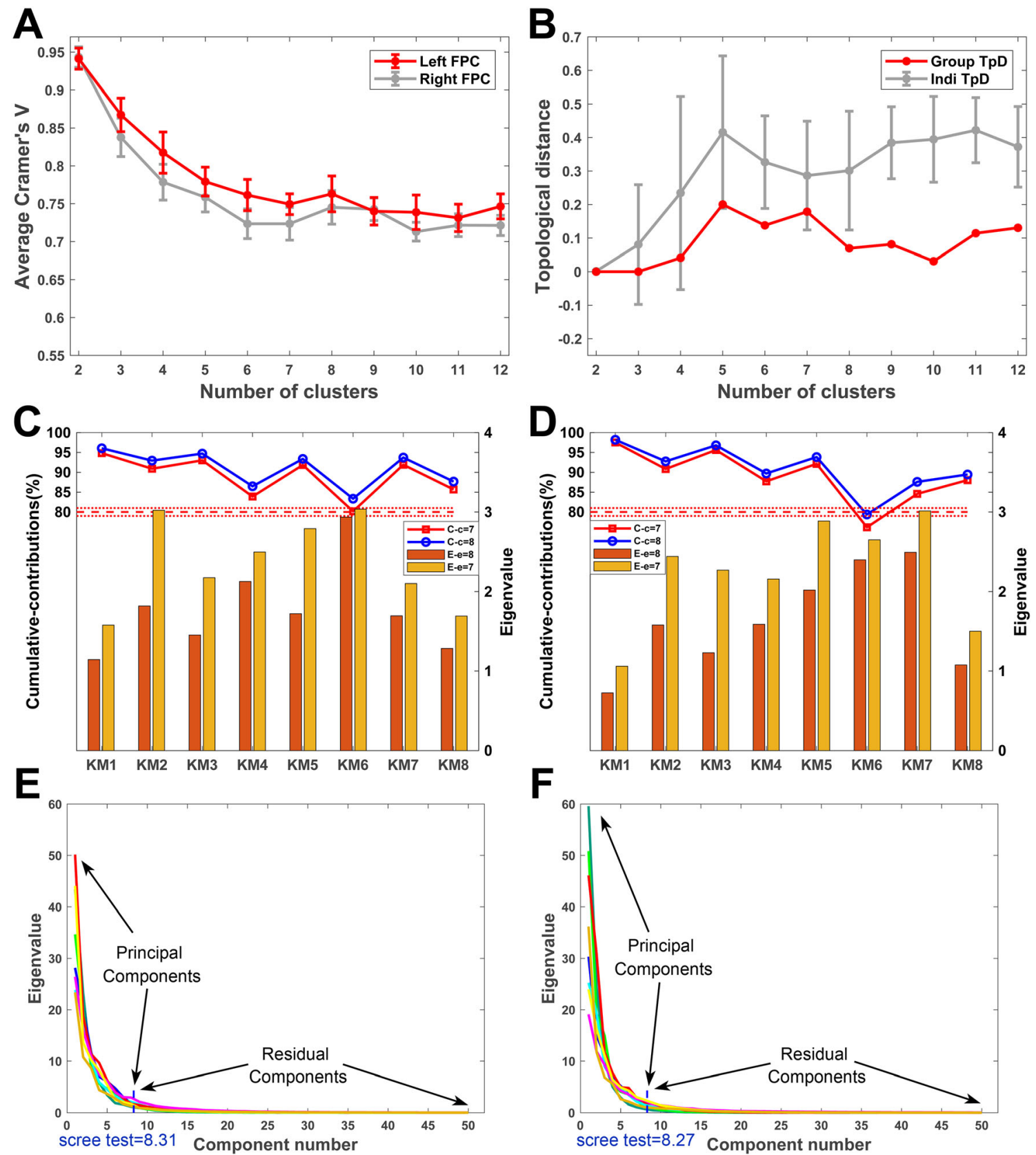

Fig. 2 Cross-validity indices of parcellation of the FPC. A, B Cluster number consistency and topological similarity indicated by the average Cramer's V (A) and TpD (B). The red/gray polyline of the average Cramer's V indicates the clustering consistency of the left/ right brain across subjects. The red/gray polyline of the $\mathrm{TpD}$ denotes the similarity of the topological arrangement of presumptive homologous regions between hemispheres and across subjects (KM1, KM2, ..., KM8) at the group/individual level. C, D Cumulative contribution

\section{Cluster C4}

A distinct cluster, $\mathrm{C} 4$, occupied the lateral orbital part of the FPC. In the coronal plane, following an anterior-toposterior direction, it was below Cluster C6 and its medial border was delimited by Cluster C3, gradually disappearing

rates and eigenvalues for the left $(\mathbf{C})$ and right $(\mathbf{D})$ hemisphere under criterion 1 and criterion 2. A comparison of the blue and red curves reveals that eight principal components (blue) are superior to seven (red). The bars represent the eigenvalues of the seventh (yellow) and eighth (brown) components. E, F Graph of principal components according to their eigenvalue sizes for the left (E) and right (F) hemispheres for all specimens.

with Cluster C6. Its lateral part was below the ps and extended medially above the ps near the interface of ps and ros from the sagittal section followed by its extension to the anterior of area 11 in the lateral orbitofrontal cortex. The connectivity of Cluster $\mathrm{C} 4$ was similar to that of cluster C3. The difference was that the former had stronger 
Fig. 3 Connectivity-based parcellation (left hemisphere) of the macaque FPC on F99 surfaces. A The subdivisions are depicted on a flat surface (right) and a fiducial surface (left) of the lateral and medial views. Each subregion is coded with a unique color and named arbitrarily $\mathrm{C} 1, \mathrm{C} 2, \ldots, \mathrm{C} 8 . \mathbf{B}$ The probability map of each FPC subarea. The color bar represents the mean probability across subjects at each voxel.
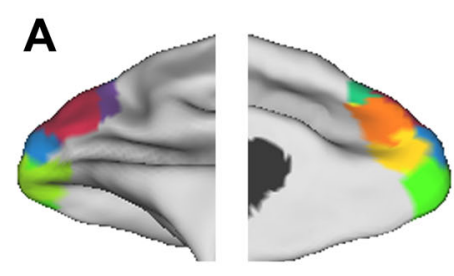

B

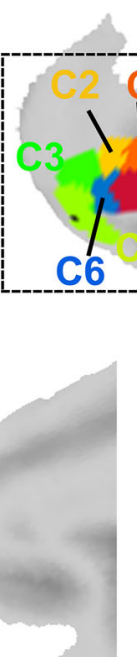

C2
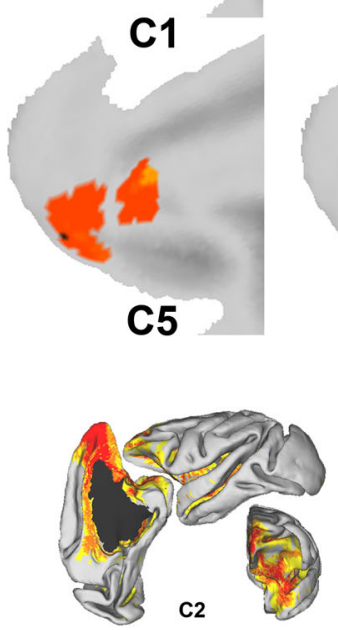

C2
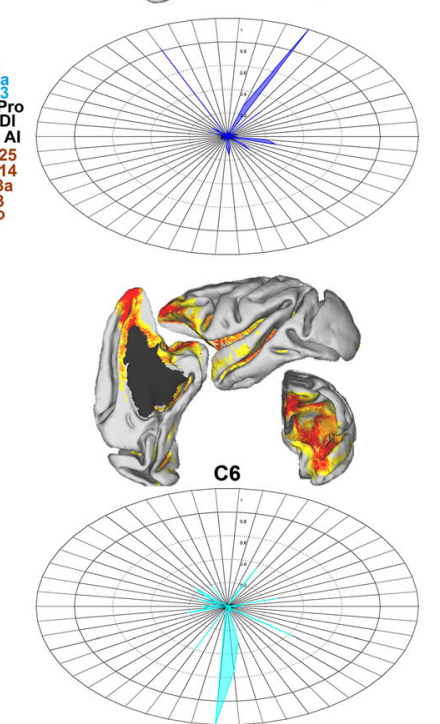
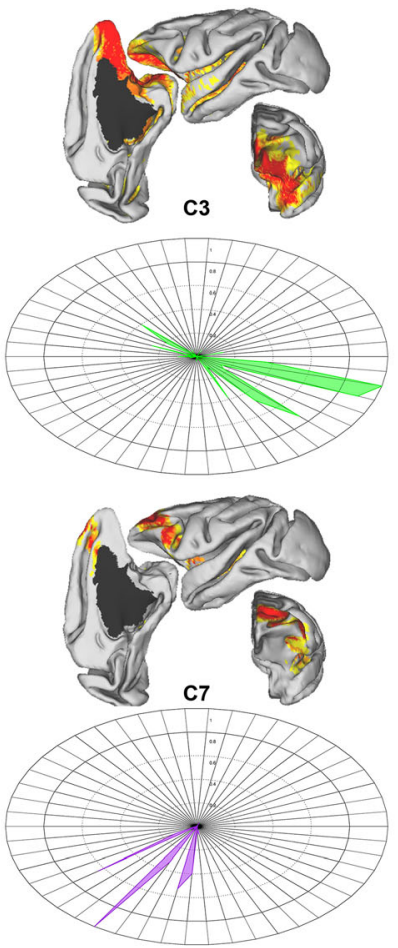

1.0

C3

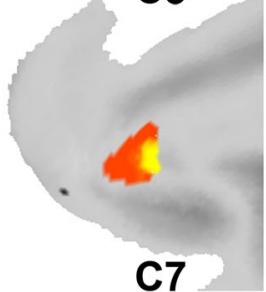

C4

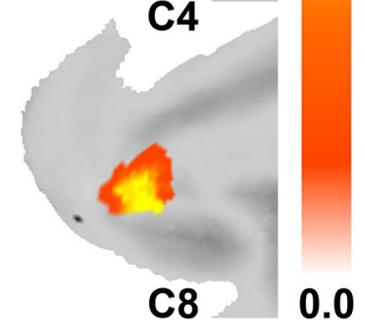

Fig. 4 Anatomical connectivity patterns between each subarea and cortical structures (left hemisphere). The connectivity of each cluster yielded by tractography-based parcellation shown in the F99 surface using Caret helps to qualitatively identify differential connections. Anatomical connectivity fingerprints quantitatively identify the differences of the connectivity patterns between each subarea and the cortical structures. For the fingerprints, we classified the connected brain regions on the periphery of the ellipse based on the different brain structure to which they belong, and display them using different color fonts (starting from area AI, and anticlockwise, the regions with different color fonts represent the insular, cingulate, occipital, temporal, frontal, and orbitofrontal cortices). Each subarea is named $\mathrm{C} 1, \mathrm{C} 2, \ldots, \mathrm{C} 8$. 

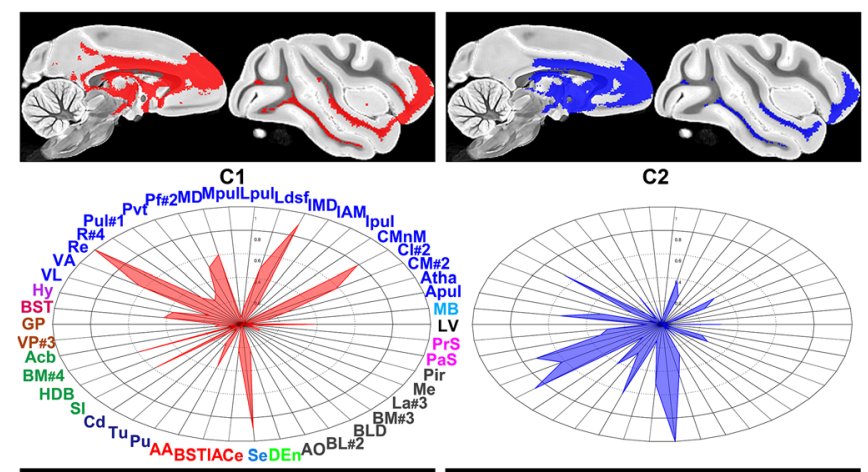

C2

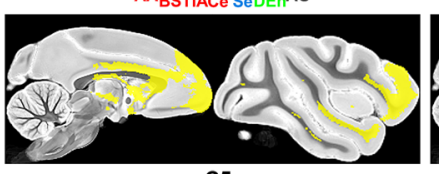

C5
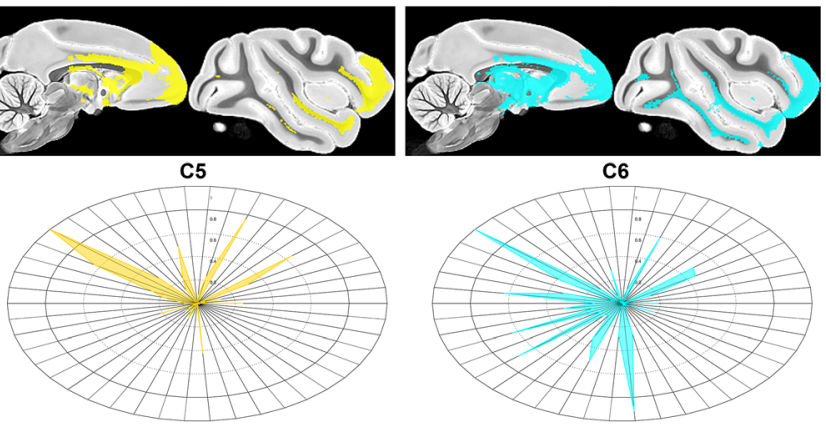

C6

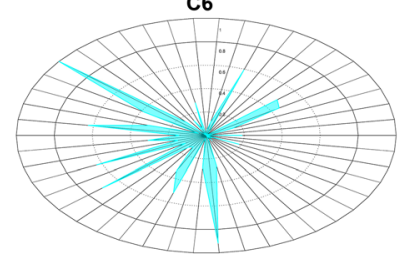

Fig. 5 Anatomical connectivity patterns between each subarea and subcortical structures (left hemisphere). Population maps of the whole brain anatomical connectivity patterns shown in CIVM space using MRIcron help to qualitatively identify differential connections, and the connection pattern of each area is colored differently. Anatomical connectivity fingerprints quantitatively identify the differences of the connectivity patterns between each subarea and the subcortical structures. For the fingerprints, we classified the connected regions on

connections with the lateral and orbital frontal cortex than the latter. For instance, stronger connections with Cluster $\mathrm{C} 4$ were detected throughout area 11, the ventral part of area 10, and the orbital proisocortex. In contrast, the connections with subcortical structures, including SI, Se, $\mathrm{BM \# 4}$, and BST, were similar but fewer than those for Cluster C3.

\section{Cluster C5}

C5 covered a small region, relative to the other subregions, in the medial dorsal part of the FPC. The distribution of Cluster C5 was mainly around the white matter above the cgs from the coronal plane. It exhibited strong connectivity with the dorsolateral frontal cortex and ProM, including areas $9 / 46 \mathrm{D}, 24 \mathrm{a}, 9 / 46 \mathrm{D}$, and the dorsal and medial parts of area 10. It also had a stronger connection with 6VR than the other subareas. Some subcortical structures, i.e. Re, IMD, VA, and $\mathrm{Cl} \# 2$, had a strong connectivity with Cluster C5.

\section{Cluster C6}

C6, which was located in the lateral middle part of the FPC, was focused around the anterior of the aspd. It lay above

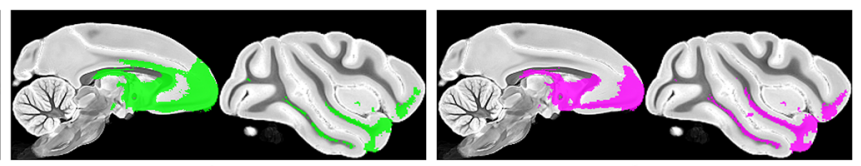

C3

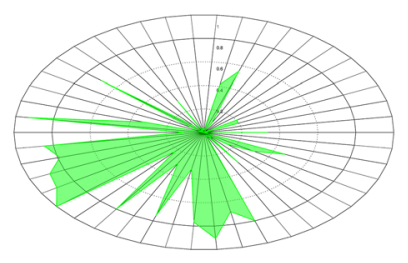

C4

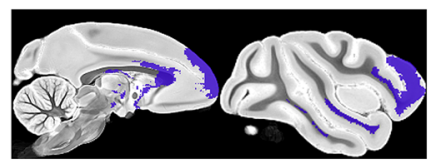

C7
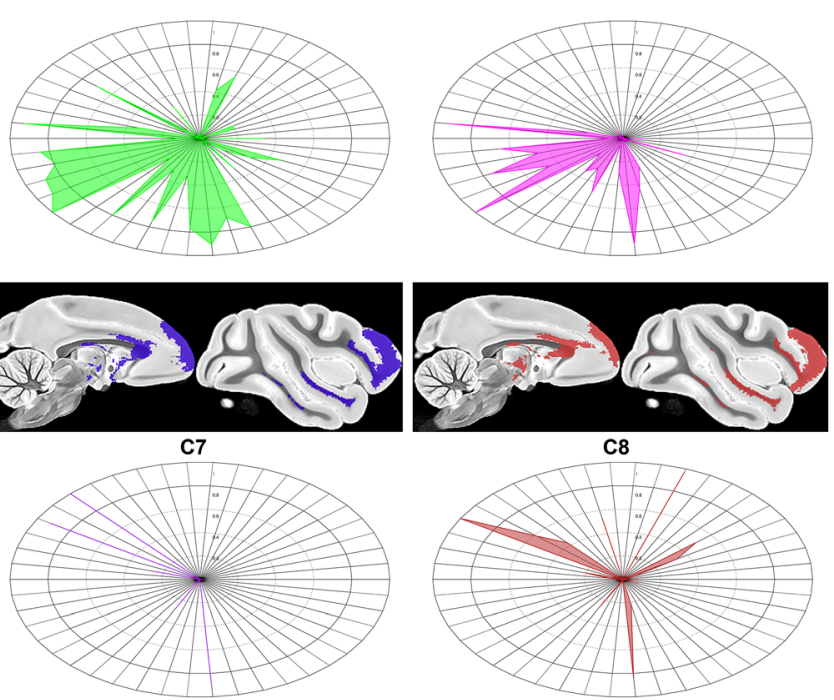

C8

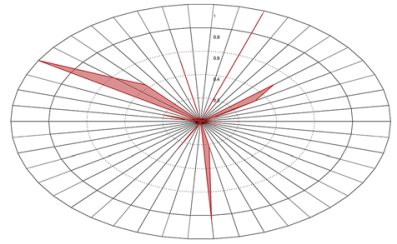

the periphery of the ellipse based on the different structure to which they belong, and display them using different color fonts (starting from area LV, and anticlockwise, the brain regions with different color fonts belong to the lateral ventricles, midbrain, hypothalamus, central subpallium, pallium, paraseptal subpallium, striatum, subpallial amygdala, subpallial septum, lateral pallium, ventral pallium, and medial pallium). Each subarea is named $\mathrm{C} 1, \mathrm{C} 2, \ldots, \mathrm{C} 8$.

the ps and a portion of this subregion extended to the medial surface. Following a medial-to-lateral direction, its superior and posterior borders were delimited by Clusters $\mathrm{C} 1$ and $\mathrm{C} 2$, then, gradually, with the disappearance of these two subdivisions, its superior border was limited by Cluster C8. This subdivision was around the rostral part of the ps and covered part of its anterior bank. Its inferior adjacent subarea was Cluster C4. Cluster C6 was mainly connected to the anterior-most frontal cortex, 46, 47, and the orbital proisocortex. With respect to the subcortical structures, it had a strong connectivity with Re, Se, SI, BM\#4, and BST.

\section{Cluster C7}

C7 occupied the dorsal lateral part of the FPC. It was delimited posteriorly by Cluster C5 near the medial surface, gradually limited by the adjacent area 9 and covered the posterior bank of the aspd following a medialto-lateral direction. The ventral extension was limited by Cluster C8. It was characterized by a very strong connectivity with the dorsal and lateral frontal lobe, including the adjacent areas of 10D, 9/46D, 9/46V, 45, 46, and 9. In addition, the subcortical structures R\#4 and VA shared a stronger connectivity seeded from area $\mathrm{C} 7$. 


\section{Cluster C8}

C8 was located in the dorsal inferior part of the lateral FPC. It covered a large part above the most anterior limb of the ps. C8 occupied a small area near the medial surface, relative to the other clusters. Following a medial-to-lateral direction, it was gradually sandwiched between $\mathrm{C} 7$ and $\mathrm{C} 6$ and extended to the superior margin of area 46D near the most lateral surface. The connectivity patterns of Clusters C7 and C8 were very similar, but the latter had stronger connections with areas 9/46V, 46, 47, and ProM.

\section{Similarity Analysis and Repeatability of Connected Brain Regions, and FPC Modularity Structure}

After the connection differences between subdivisions were determined, the inter-individual correlation index revealed a high level of connection similarity for the eight clusters (Fig. 6A and S4A), which suggests that the connectivities of parcellation results are very consistent among the eight subjects. Using the CoCoMac data, we identified the areas derived from our connectivity data and theirs that were derived from the axonal tracer projections that originate in the FPC, and calculated an $88.24 \%$ coherence (Fig. 6B and S4B), which to some extent suggests repeatability of the connected areas and the reliability of our parcellation results.

Then, using the improved hierarchical clustering method, the eight subdivisions were grouped into three contiguous boundary connectivity families: medial FPC (MF; C1, C2, C3), dorsolateral FPC (DLF; C5, C6, C7, C8), and lateral orbital FPC (LOF; C4) (Fig. 6C and S4A). A dendrogram was constructed using the standardized Euclidean distance (seuclidean) and average linkage (average) method because this method led to the most faithful representation of the original distances based on their highest cophenetic coefficients (left brain, 0.92; right brain, 0.94). As a mediator of network modularity in the macaque [66], these FPC subregions connected to other regions with inter-area coordination; in detail, the MF mainly connected the "medial" brain network, the DLF connected to most of the regions of the dorsal brain network, and the LOF mainly connected to the orbital brain regions.

Further, based on the anatomical connections of each subarea and the modular analysis, we found that these subareas were collaboratively involved in the DMN, SIN (Fig. 6D and S4D), and metacognition network. First, previous studies have revealed that the macaque FPC is functionally correlated with the DMN $[22,67]$. We found that the DLF, in conjunction with an extension to the medial part $(\mathrm{C} 1, \mathrm{C} 2)$, had strong connection probabilities to areas of the DMN. In particular, areas C1, C5, and C7 had a strong connection with the DMN core. Second, our results revealed that the orbital and medial FPC play an important role in connectivity to the SIN, and the medial FPC showed a stronger connection than other parts of the FPC with the exclusively SIN (ESIN) [23]. The medial FPC (areas C1, $\mathrm{C} 2$, and $\mathrm{C} 5$ ) had a strong connection with areas $32,10 \mathrm{M}$, 24b, 9M, 44, 6VR, and 24b. Areas C3, C4, and C6 showed a strong connection with areas 14, 47, OPro, 11L, 10o, R36, ST1, ST2, TAa, TPPro, Cd. In addition, the dorsal FPC had strong connections with the regions involved in metacognition; in particular, subareas $\mathrm{C} 5, \mathrm{C} 7$, and $\mathrm{C} 8$ had a strong connection with the cortical region anterior to the pspd (aPSPD) and metamemory processing regions, including areas 9L, 9/46D, 46D, 8A, and 6VR. Besides, the regions of metacognitive performance for remote memory have mainly been identified in the dorsal frontal lobe; dorsal FPC showed strong connections with them. These dorsal FPC subareas mainly connected the memory retrieval regions, including the anterior bank of the frontal cortex (area 45B) and area $9 / 46 \mathrm{~V}$, but we found no connections between the FPC and the parietal cortex. Further, subarea C5 had a strong connection with the cortical network module of retrieval-related regions (area DI and 6VR).

\section{Discussion}

In this study, we performed a tractography-based parcellation and divided the macaque FPC into eight subregions, and then elucidated the anatomical connectivity patterns of the macaque FPC at the subregional level, and finally explored the modularity of the eight subareas. To more fully elucidate the reasonability of our parcellation results, we compared our parcellation and anatomical connectivity results from DTI data with previous relevant studies from a variety of perspectives, including the overlap between our boundaries and those from other parcellation results and between our connection results and those that were obtained using tracer injections.

First, the eight subregions were distinguished by different boundaries, some of which coincided well with those of other parcellation studies [30,68]. The parcellation results of the macaque FPC have varied over the past few decades. Initially, the FPC was recognized as a single area; then it was subdivided into two areas by Carmichael, Price [20], who thought that the medial part of the FPC had a homogeneous granular structure. Subsequently, however, different connections of subareas in this area were found $[19,69,70]$. Here, we found that the orbital FPC can be further subdivided into two subregions $(\mathrm{C} 3$ and $\mathrm{C} 4)$ and area $10 \mathrm{~m}$ of Carmichael, Price [20] can be subdivided into a number of subregions (Fig. 7A). In addition, the dorsolateral boundary (G-b3) and another lateral boundary (G- 


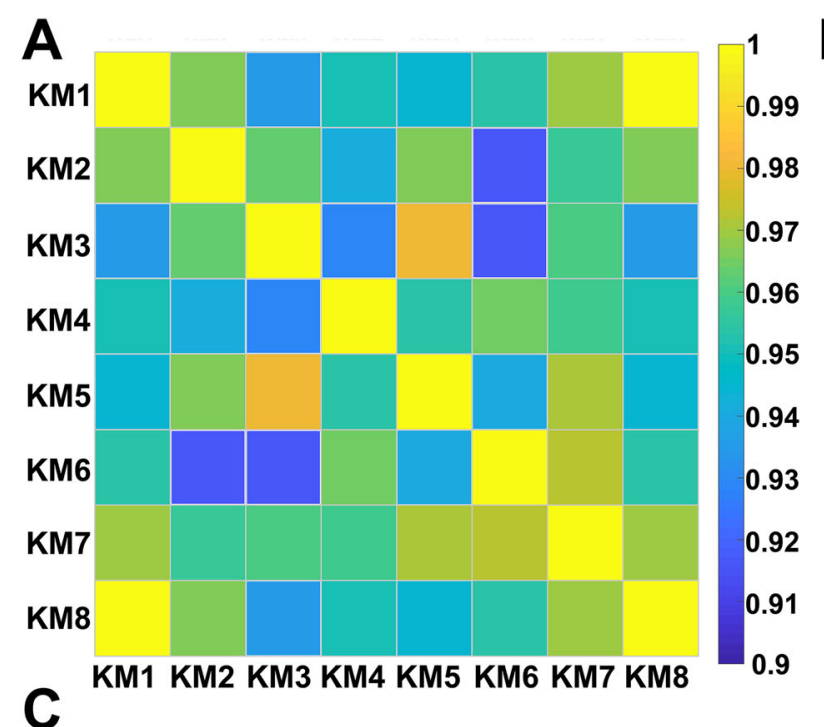

B
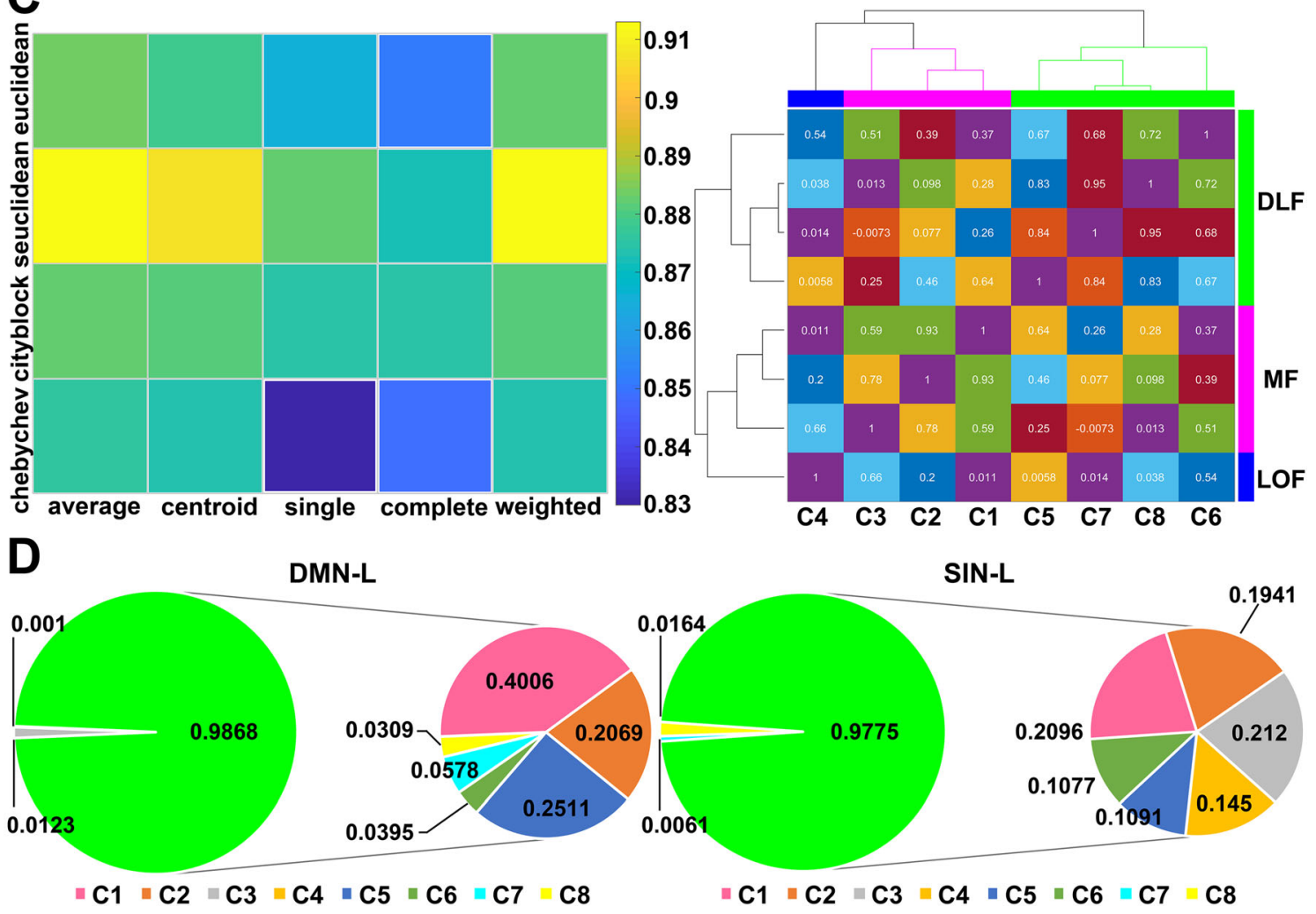

Fig. 6 Similarity analysis and repeatability of connected brain regions, and modularity analysis (left hemisphere). A The connectivity similarity matrix for all the subareas across different subjects (KM1, KM2, ..., KM8). B Consistency comparison between tracer projections of CoCoMac and the anatomical connections identified by our study. The areas around the outside edges of the ellipse are the tracer results from $\mathrm{CoCoMac}$; the areas marked in orange are the anatomical connections we found, and the gray means that we did not find these connections. C Optimization cophenetic coefficient parameter selection, connectivity similarity matrix, and dendrogram

b4) above the rostral ps in the macaque frontal cortex in another parcellation result [68] have positions similar to the boundaries between $\mathrm{C} 7$ and $\mathrm{C} 8$ (b3) and between $\mathrm{C} 8$ and

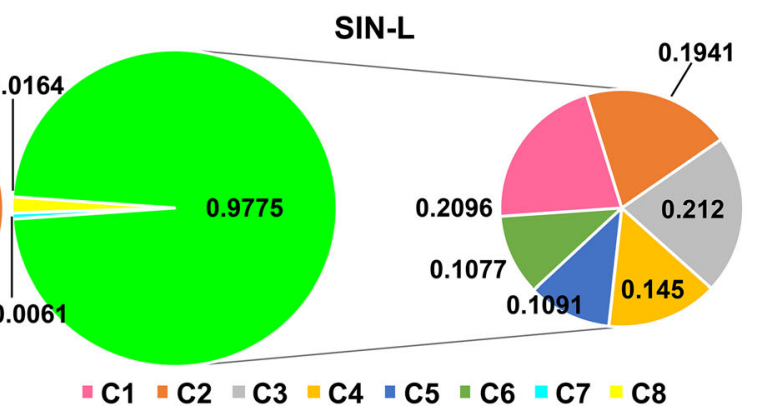

constructed on the basis of connectivity similarity for all the clusters. D Diagrammatic summary of the primary connections between the subdivisions and the regions of different functional networks. The connection probabilities involved in different functional networks for each subarea are normalized in this display. Each block of the pie chart represents the anatomical connection after normalization between each subarea and the regions of different functional networks. The green circles on the left represent the sum of the primary connections on the right.

C6 (b4), respectively, in our parcellation results. The lateral boundary (b4) and another boundary (b5) along the anterior extension of the ros were approximatively the 
same in another study (C-b4 and C-b5) [30]. These findings, on the one hand, support the reliability of previous boundary parcellations. On the other hand, they imply that the CBP technique can identify even more possible subdivisions. In particular, the FPC, a thick, highly granular cortex, has gradual differences that make it difficult to further parcellate additional subareas using traditional methods [71].

Second, our anatomical connection results are in good agreement with those of the tracer injection experiments, which are the gold standard for assessing connectivity. This revealed a potential concordance of the relationship between the connectional and microstructural properties of brain regions. In addition to the consistency comparisons with CoCoMac, comparisons with other tracer experiments that have more narrowly defined injection sites well support our anatomical connection and parcellation results. It is particularly worth noting that the tracer injection results of Saleem, Miller, Price [26] provide good evidence for our segmentation results. In that study, the $10 \mathrm{mr}$ injection site covered approximately the same area as our dorsal subareas $(\mathrm{C} 1, \mathrm{C} 2, \mathrm{C} 6$, and $\mathrm{C} 8)$, and the orbital subareas ( $\mathrm{C} 3$ and $\mathrm{C} 4)$ correspond to a different injection site (10o). For the cortical connections, we found rich intrinsic connections between the FPC and the prefrontal cortex (PFC) and distinct extrinsic connections with these regions outside the $\mathrm{PFC}$, a finding which is consistent with the tracer projection results of Saleem et al. (see Table 2). The intrinsic connections between the orbital subareas and areas $11 \mathrm{~m}, 12 \mathrm{o}, 12 \mathrm{l}, 10 \mathrm{~m}, 10 \mathrm{o}, 46 \mathrm{~d}, 32,13 \mathrm{~b}, 14 \mathrm{r} / \mathrm{c}$, and AI, as well as the extrinsic connections with areas ST1, ST2, and ST3 are consistent with the results of Saleem et al. In addition, the dorsal subareas have intrinsic connections with areas 46v, 45a, 45b, 8AD, 10mr, 10o, 9m, 91, 46d, $13 \mathrm{~m}, 12 \mathrm{o}, 10 \mathrm{mc}, 11 \mathrm{~m}, 32,25, \mathrm{AI}, 13 \mathrm{a} / \mathrm{b}$, and $14 \mathrm{r} / \mathrm{c}$ and extrinsic connections with areas ST1, ST2, ST3, 24a, 24b, 24c, 23, v23, and 29/30. These intrinsic and extrinsic connections were also found by Saleem et al. In addition, we also found no connections between the FPC and the parietal cortex, which is consistent with the conclusions of Saleem et al. [26], Petrides and Pandya [72], and Rushworth et al. [6]. More comparisons of cortical connections and subcortical connections further suggest good consistency between our anatomical connections and other tracttracing studies (details in Table 2 and Table 3).

In addition, the topological modules of brain networks often consist of anatomically neighboring cortical areas, and exploring the brain modular structure can heuristically

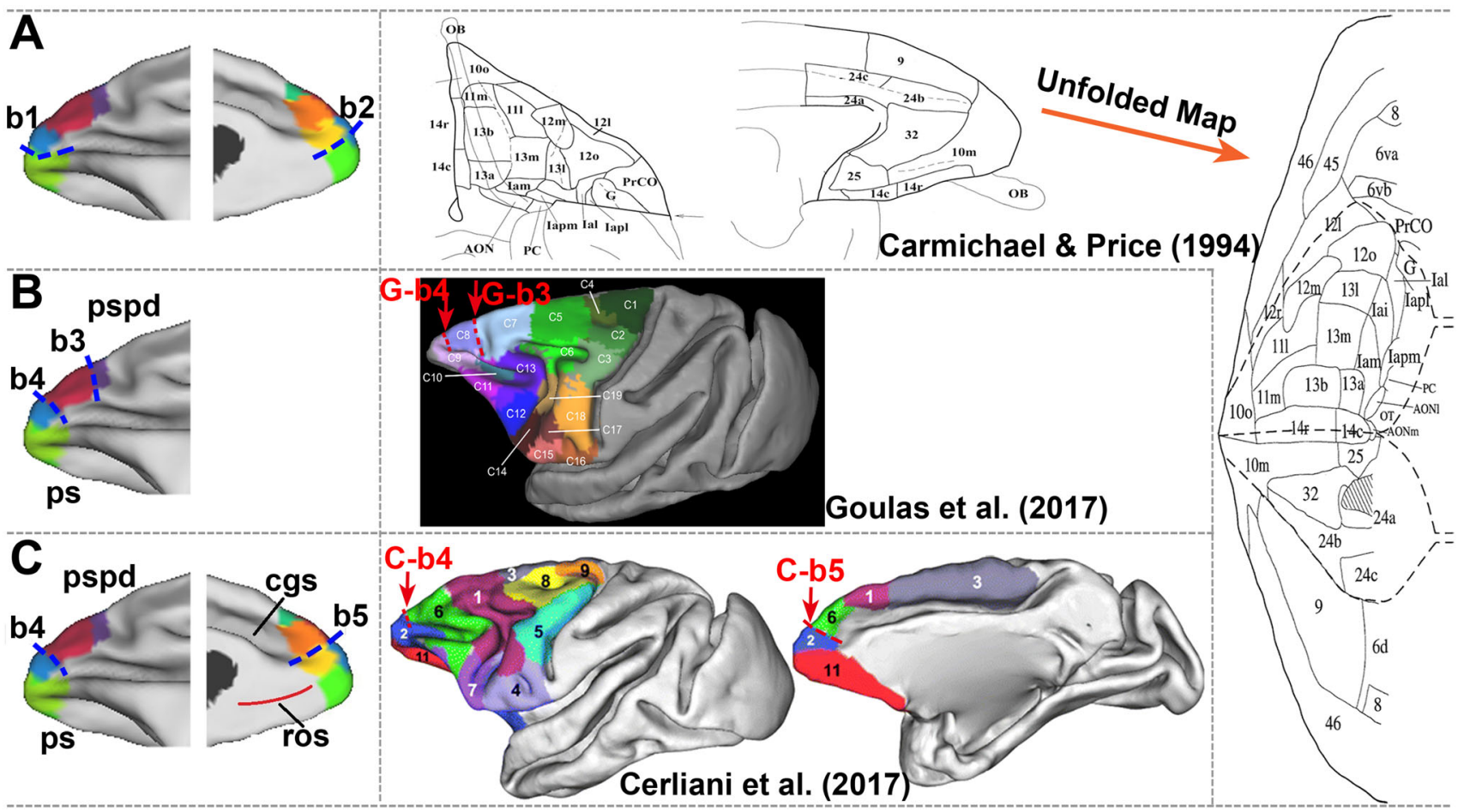

Fig. 7 Side-by-side comparison between the results of the groupaveraged, connectivity-based parcellation described in the current study (left) and the macaque maps (right) from other studies. A The subareas of Carmichael and Price (1994) can be further subdivided. B The lateral boundary $\mathrm{b} 1$ and medial boundary b2 distinguish the lateral subareas $\mathrm{C} 4$ and $\mathrm{C} 6$ and the medial subareas $\mathrm{C} 2$ and $\mathrm{C} 3$, respectively. Other lateral boundaries, $\mathrm{b} 3$ and $\mathrm{b} 4$, corresponding to the red boundaries of Goulas et al. (red arrow, G-b3 and G-b4), distinguish the lateral subareas $\mathrm{C} 7$ and $\mathrm{C} 8$ and subareas $\mathrm{C} 8$ and C6, respectively. $\mathbf{C}$ The medial boundary b5, corresponding to the medial red boundary of Cerliani et al. (red arrow, C-b4 and C-b5), distinguishes the medial subareas $\mathrm{C} 1$ and $\mathrm{C} 2$. 
Table 2 Consistent cortical connections from our tractography compared with the data acquired in previous studies using tracer injection.

\begin{tabular}{|c|c|c|c|c|}
\hline Experiments & Cases/case no. & Injection site(s) & $\begin{array}{l}\text { Corresponding } \\
\text { subregions }\end{array}$ & Projections found \\
\hline $\begin{array}{l}\text { Barbas and } \\
\text { Mesulam } \\
{[73]}\end{array}$ & Case V, (HRP) & $\begin{array}{l}\text { Rostral principalis } \\
\text { region (rostral } \\
46 \text { and } 10)\end{array}$ & $\mathrm{C} 6, \mathrm{C} 8, \mathrm{C} 7$ & $\begin{array}{l}\text { Dorsal and medial parts of the FPC, } 14,46,12,11,9 / 46 \mathrm{D} \text {, } \\
\text { R36, TTPAl, ST1, ST2, ST3, TAa }\end{array}$ \\
\hline \multirow{4}{*}{$\begin{array}{l}\text { Barbas and } \\
\text { Pandya } \\
{[27]}\end{array}$} & Case 1, (isotope injection) & OPro & & $\mathrm{C} 3, \mathrm{C} 4, \mathrm{C} 6, \mathrm{C} 7$ \\
\hline & Case 3, (isotope injection) & 14,13 & & $\mathrm{C} 3, \mathrm{C} 4$ \\
\hline & Case 4, (isotope injection) & Orbital area 12 & & \\
\hline & Case 6, (isotope injection) & 46 & & \\
\hline $\begin{array}{l}\text { Barbas et al. } \\
\text { [74] }\end{array}$ & Case ARb, (FB) & Medial area 10 & $\mathrm{C} 1, \mathrm{C} 5$ & $9,46,24,32,12,14,11,25,8,13$, OPro, TTPAl, TPPro \\
\hline \multirow{2}{*}{$\begin{array}{l}\text { Germuska } \\
\text { et al. }[12]\end{array}$} & Cases BA, (BDA) & & $\mathrm{C} 4$ & ST1, ST2, TTPA1, TPPro \\
\hline & Cases BC, BF, (BDA) & & $\mathrm{C} 7, \mathrm{C} 8$ & ST1, ST2, ST3 \\
\hline $\begin{array}{l}\text { Parvizi } \\
\text { et al. }[75]\end{array}$ & $\begin{array}{l}\text { M1-BDA-23B, M1-FB-31, } \\
\text { M2-BDA-23a/b, M3- } \\
\text { BDA-29/30(23a) }\end{array}$ & $\begin{array}{l}23 b, 31,23 a, 23 b \\
29 / 30(23 a)\end{array}$ & & Dorsal and dorsomedial parts of the FPC $(\mathrm{C} 1, \mathrm{C} 2, \mathrm{C} 5, \mathrm{C} 8)$ \\
\hline \multirow{3}{*}{$\begin{array}{l}\text { Petrides and } \\
\text { Pandya } \\
{[76]}\end{array}$} & Case $4,(\mathrm{DY})$ & Lateral area 9 & & Dorsal subareas $(\mathrm{C} 5, \mathrm{C} 7)$ \\
\hline & Case 6, (FB) & $9 / 46 \mathrm{~d}$ & & $\mathrm{C} 1, \mathrm{C} 5$ \\
\hline & Case $8,(\mathrm{FB})$ & $\begin{array}{l}\text { Dorsal area } 46 \\
\text { (close to FPC) }\end{array}$ & & FPC \\
\hline \multirow{2}{*}{$\begin{array}{l}\text { Petrides and } \\
\text { Pandya } \\
\text { [72] }\end{array}$} & Case 1, (isotope injection) & Area 10 & FPC & $\begin{array}{l}\text { 9, 46, 32, } 11,13,14,8 \text { A, 47/12, 45, 23, 24, 25, 30, OPro, } \\
\text { ST1, ST2, ST3, TAa, TPPro, PaI, AI, DI }\end{array}$ \\
\hline & Case 2, (isotope injection) & $\begin{array}{l}\text { Ventral and orbital } \\
\text { area } 10\end{array}$ & $\mathrm{C} 4$ & $46,10,11,14,47 / 12,14,13,32,25$, ТАа \\
\hline \multirow[t]{2}{*}{$\begin{array}{l}\text { Saleem } \\
\text { et al. }[26]\end{array}$} & OM19 (FB) & $10 \mathrm{o}$ & $\mathrm{C} 3, \mathrm{C} 4$ & $\begin{array}{l}\text { 47/12(12o,12l), 10mr, 10o, 46(46d), 13(13b), 10mc, } 11 \mathrm{~m} \text {, } \\
\text { 14(14r/c), 32, AI, ST1, ST2, ST3 }\end{array}$ \\
\hline & OM69 (FB), OM64 (FB) & $10 \mathrm{mr}$ & $\mathrm{C} 1, \mathrm{C} 2, \mathrm{C} 6, \mathrm{C} 8$ & $\begin{array}{l}46(46 \mathrm{~d}, 46 \mathrm{v}, 46 \mathrm{f}), 45(45 \mathrm{a}, 45 \mathrm{~b}), 8 \mathrm{~A}(8 \mathrm{Ad}), 10 \mathrm{mr}, 10 \mathrm{o}, \\
10 \mathrm{mc}, 9(9 \mathrm{~d}, 9 \mathrm{~m}), 13(13 \mathrm{~m} / 1,13 \mathrm{a}, \mathrm{b}), 47 / 12(12 \mathrm{o}), 11 \mathrm{~m}, \\
14(14 \mathrm{r} / \mathrm{c}), 32, \text { AI, 25, ST1, ST2, ST3, 24a, 24b, 24c, 23, } \\
29,30\end{array}$ \\
\hline
\end{tabular}

facilitate functional studies of localized areas and help to understand brain mechanisms $[62,63,83]$. Our results from the hierarchical clustering for the connectivity-based parcellation revealed that the different subregions collaborate to connect different functional networks.

We found that the orbital FPC connected with most of the regions of the "orbital" prefrontal network; the medial FPC mainly linked to the "medial" prefrontal network, which is consistent with the descriptions of previous network partitions [84]. Subsequently, eight subareas are collaboratively involved in the DMN, SIN, and metacognition network. The FPC has a functional involvement with the SIN [23, 85], and the medial frontal regions around the rostral tip of the ACC show significant activity during interactive social communication [86]. Correspondingly, we found that area $\mathrm{C} 1$, which is located around the rostral tip of the ACC, had a strong connection with the regions of the SIN. The theory of mind (ToM) and the DMN intersectional regions in the human brain have a fairly plausible homology and locations similar to the ESIN regions of the macaque brain. These macaque areas are in locations similar to the DMN and ToM intersectional regions in humans and share anatomical features with the human ToM and ESIN; these findings are confirmed by our results. With respect to the third function, metacognition, in macaque monkeys only the bilateral FPCs are enlisted for the metacognitive evaluation of non-experienced items, the dorsal FPC is only significantly correlated with metacognitive performance with respect to non-experienced items and serves as the neural substrate for awareness of one's own ignorance in macaques [24]. The FPC is functionally connected with the aPSPD, which is essential for the metacognitive judgment of remote memory; in particular, there is a strong resting-state functional connectivity with area 9 that is related to metamemory processing [58]. Here, we found anatomical connections between the FPC and the aPSPD with particularly strong connections with area 9. For retrieval of remote memory, the FPC may be involved in metacognitive processing. Anatomical connections between the FPC and area 9 also 
Table 3 Consistent subcortical connections from our tractography compared with the data acquired in previous studies using tracer injection.

\begin{tabular}{|c|c|c|c|c|}
\hline Experiments & Cases/case no. & Injection site(s) & $\begin{array}{l}\text { Corresponding } \\
\text { subregions }\end{array}$ & Projections found \\
\hline \multirow[t]{6}{*}{ An et al. [69] } & Case OM36, (BDA) & $10 \mathrm{~m}$ & $\mathrm{C} 1, \mathrm{C} 2$ & dorsolateral midbrain PAG \\
\hline & Case OM38, (BDA) & $10 \mathrm{o}$ & $\mathrm{C} 3$ & \\
\hline & Case OM32, (FB) & Ventrolateral midbrain PAG & & $\mathrm{C} 1, \mathrm{C} 2, \mathrm{C} 5, \mathrm{C} 6, \mathrm{C} 7, \mathrm{C} 8$ \\
\hline & Case OM35, (FB) & Dorsolateral midbrain PAG & & \\
\hline & Case OM36, (FB) & Rostral dorsolateral midbrain PAG & & \\
\hline & Case OM36, $(\mathrm{CTb})$ & Lateral midbrain PAG & & \\
\hline \multirow{2}{*}{$\begin{array}{l}\text { Ferry et al. } \\
\text { [77] }\end{array}$} & Case OM38, (BDA) & $10 \mathrm{~m}$ & $\mathrm{C} 1, \mathrm{C} 2$ & $\mathrm{Cd}, \mathrm{Acb}$ \\
\hline & Case OM38, (BDA) & $10 \mathrm{o}$ & $\mathrm{C} 3, \mathrm{C} 4$ & $\mathrm{Cd}, \mathrm{Acb}, \mathrm{Pu}$ \\
\hline \multirow{2}{*}{$\begin{array}{r}\text { Ghashghaei } \\
\text { et al. }[78]\end{array}$} & Case BD_R_BDA & BM\#3, BL\#2, BLD, $\mathrm{Me}, \mathrm{Ce}$ & & $\mathrm{C} 1, \mathrm{C} 2, \mathrm{C} 3, \mathrm{C} 4, \mathrm{C} 6$ \\
\hline & Case BD_L_BDA & & & \\
\hline \multirow{2}{*}{$\begin{array}{l}\text { Hsu and Price } \\
\text { [79] }\end{array}$} & Case OM74, (FR) & $10 \mathrm{~m}$ & $\mathrm{C} 1, \mathrm{C} 3$ & MITN, Re, CM\#2, CMnM, Cl\#2 \\
\hline & Case OM66, (FR) & & & \\
\hline \multirow{3}{*}{$\begin{array}{l}\text { Ongur et al. } \\
\text { [70] }\end{array}$} & Case OM26, (FB) & Lateral hypothalamus & & FPC $(10 \mathrm{~m}, 10 \mathrm{o})$ \\
\hline & Case OM27, (FB) & Ventromedial hypothalamic nucleus & & \\
\hline & Case OM37, (FB) & Anterior hypothalamus & & \\
\hline $\begin{array}{l}\text { Petrides and } \\
\text { Pandya [72] }\end{array}$ & $\begin{array}{l}\text { Case 1, (isotope } \\
\text { injection) }\end{array}$ & Area 10 & FPC & $\begin{array}{l}\mathrm{Lv}, \mathrm{Cd}, \mathrm{Pu} \text {, thalamus, Pul\#1, IAM, IMD, } \\
\text { Hy, amygdala, BL\#2, BLD, BM\#3, } \\
\text { hypothalamus }\end{array}$ \\
\hline $\begin{array}{l}\text { Rempel- } \\
\text { Clower and } \\
\text { Barbas [80] }\end{array}$ & Case SF, (HRP) & Dorsal area 10 & C6, C8 & hypothalamus \\
\hline \multirow[t]{4}{*}{$\begin{array}{l}\text { Romanski } \\
\text { et al. }[81]\end{array}$} & $\begin{array}{l}\text { Case Fig. 7C, } \\
\text { (WGA-HRP) }\end{array}$ & FPC & $\mathrm{C} 1, \mathrm{C} 2, \mathrm{C} 4, \mathrm{C} 6$ & MPul, Pul\#1 \\
\hline & $\begin{array}{l}\text { Case 1, (WGA- } \\
\text { HRP) }\end{array}$ & Medial pulvinar & & $\mathrm{C} 1, \mathrm{C} 4$ \\
\hline & $\begin{array}{l}\text { Case 2, (WGA- } \\
\text { HRP) }\end{array}$ & Central/lateral PM & & $\mathrm{C} 4$ \\
\hline & $\begin{array}{l}\text { Case 3, (WGA- } \\
\text { HRP) }\end{array}$ & $\begin{array}{l}\text { Medial region of the PM (intruded on } \\
\text { caudal, medial regions of the } \\
\text { mediodorsal nucleus) }\end{array}$ & & $\mathrm{C} 1, \mathrm{C} 3$, \\
\hline Cho et al. [82] & $\begin{array}{l}\text { Cases J12FR, } \\
\text { J12LY, J16LY, } \\
\text { J8LY, J12FS }\end{array}$ & BM\#4 & & $\mathrm{C} 1, \mathrm{C} 2, \mathrm{C} 3, \mathrm{C} 4$ \\
\hline
\end{tabular}

$F B$ fast blue, $D Y$ diamidino yellow, $H R P$ horseradish peroxidase, $B D A$ biotinylated dextran amine, $C T b$ cholera toxin subunit $\mathrm{B}, W G A-H R P$ wheat germ agglutinin-horseradish peroxidase, $F R$ fluoro-ruby, $L Y$ Lucifer yellow, $F S$ fluorescein.

suggested that these two regions work cooperatively to support metacognitive judgments in ecological situations. These findings suggested a consistent relationship between functional activation and connectivity fingerprints [87]. Previous studies have reported that the corresponding memory-related regions between humans and macaques have not been established. Consistent with the previous studies [6, 26, 72], we found no connections between the FPC and the parietal regions. Notably, the medial subareas $\mathrm{C} 1$ and $\mathrm{C} 2$ had rich connections with other regions of different functional networks, a finding which suggests that these two subareas play an important role in coordinating other subareas to participate in different network functions.
To sum up, the above comparative findings validate the reliability of our parcellation results and indicate that anatomical connections and tracer-injection studies provide consistent results. We need to mention that, although different studies $[30,68]$ have often disagreed about the definition of the borders of the subareas in the macaque frontal cortex, if a boundary near a similar position was found in other studies, we were more convinced of its authenticity. The anatomical connections estimated from diffusion tractography may susceptible to false positives (the tracking of pseudo-pathways) and false negatives (the inability to track pathways that have been found), and do not furnish the level of detail of the gold standard based on 
invasive tract-tracing techniques, but DTI has proven to be an indispensable method and can offer invaluable insights for neuroscience [88] and neuroanatomy [89, 90], including the discovery of new pathways [31], the description of whole-brain connectivity information [32], and the refinement of brain regions [28].

The methods used in the current study are discussed below, along with their advantages, disadvantages, and problems of validation. First, previous studies have suggested that the CBP method can yield more fine-grained parcellations than traditional cytoarchitectonic mapping, and compared with other neuroimaging methods, it has the pivotal strength to actually map distinct brain regions without sample size restriction [33, 91]. Second, to some extent, challenges were raised in CBP studies because of the inter-individual variability, which made it difficult to relate the anatomical connectivity patterns of a region to its functional roles [29]. Third, in this study, the efficiency of the parcellation framework based on CBP and the parameters for parcellation have been validated by many studies [33, 43, 48, 92]. In general, it is worth noting that all the parameters must be reasonable, which means that they cannot be uncommon extreme values, otherwise wrong results will be tracked. One of the effective verification methods is to compare the results of the anatomical connections with those of tracer injection [93]. There are many parameters in tractography, and all of these affect the results of fiber tracking to different degrees, more or less (i.e., number of samples, distance correction, step length, curvature, exclusion mask, track style, number of steps per sample ...). In particular, some studies have reported the effects of these parameters [94-99]. However, in the current study, we mainly set two parameters (number of samples $=15000$; step size $=0.2 \mathrm{~mm}$ ), other parameters are based on the default values. All these parameters were based on previous studies [33, 42] and the official instructions of FSL. Tournier et al. have revealed that the dispersion in tractography is dependent on the step size; small step sizes reduce the spread of probabilistic tracking results [98]. Therefore, to explore the sensitivity of the parcellation results to the number of samples, here we used different samples of tractography and carried out repetitive parcellation experiments on the macaque FPC (left brain, see details in supplementary materials; the details of the experiment and the stability validation of the parcellation method have also been described in our previous study [42]). In addition, for CoCoMac, there are still some challenges in automatically extracting data from published studies [100]. The results of tracer experiments such as those obtained from the CoCoMac database or other databases [101] are not only limited to invasive approaches to some extent, but also limited to the number of samples and lack of consideration of individual variation. The data may also miss tiny pathways and produce slightly different projections caused by individual differences, so we agree that the combination of tractography and the tracer injection results is an effective and complementary way to assess brain connectivity, which is crucial for accurately mapping structural connectivity [102]. Advances in MRI have made it increasingly feasible to calculate their connections [93], and DTI tractography is capable of providing inter-regional connectivity comparable to neuroanatomical connectivity [103]. In the current study, the consistency of the connectivity comparison with other relevant studies increases the confidence in the structural connectivity of the macaque FPC and is important for studying FPC-related networks of brain functions and their disorders [104, 105]. In future, we plan to conduct a tracer injection based on the parcellation results in the current study to explore the detailed connectivity of each subregion by a quantitative cytoarchitectonic analysis and evaluate the degree of consistency between anatomical connections and tracer injections in the same subjects. Furthermore, we have released the detailed parcellation pipeline and will then apply it to the whole macaque brain to obtain a finegrained macaque brain atlas.

Besides, compared with other methods, the framework of CBP provided in the current study not only inherited the advantages of other classical CBP methods [48, 50] but also improved them in selecting the optimal clustering scheme and making them more adaptable to non-human primates [42]. The results of the modularity could heuristically facilitate functional studies of localized areas and exploration of the connectivity patterns of different functional networks is important for understanding brain mechanisms and evolution. Furthermore, based on previous studies [68], our proposed hierarchical clustering algorithm can automatically select the best clustering parameters to generate an optimal clustering result by calculating and comparing all the cophenetic correlation coefficients, which is helpful for users to group the clusters into different broad connectivity families.

\section{Conclusions}

In the present study, we used a CBP scheme for the macaque FPC and divided it into eight distinct subareas. As a powerful analytical framework, CBP not only reveals the spatial distribution of cytoarchitectural boundaries but also provides supplementary information related to the organization of anatomical and different functional networks among brain regions.

Furthermore, by using a hierarchical clustering algorithm, we identified the modularity of the bilateral FPC and found synergy related to the DMN, SIN, and metacognition 
network among the subdivisions. We hope that all of the above information is helpful for understanding the anatomy and circuitry of related regions and can facilitate the use of available knowledge in FPC-related clinical research, especially in understanding the dysfunctions caused by complex diseases.

Acknowledgements We thank E. Rhoda and Edmund F. Perozzi for constructive comments on the manuscript and great help with the English language. This work was supported by the National Natural Science Foundation of China (91432302 and 31620103905 ), the Science Frontier Program of the Chinese Academy of Sciences (QYZDJ-SSW-SMC019), the National Key R\&D Program of China (2017YFA0105203), Beijing Municipal Science and Technology Commission (Z161100000216152, Z161100000216139, Z181100001518004 and Z171100000117002), the Beijing Brain Initiative of Beijing Municipal Science and Technology Commission (Z181100001518004), and the Guangdong Pearl River Talents Plan (2016ZT06S220).

Open Access This article is licensed under a Creative Commons Attribution 4.0 International License, which permits use, sharing, adaptation, distribution and reproduction in any medium or format, as long as you give appropriate credit to the original author(s) and the source, provide a link to the Creative Commons licence, and indicate if changes were made. The images or other third party material in this article are included in the article's Creative Commons licence, unless indicated otherwise in a credit line to the material. If material is not included in the article's Creative Commons licence and your intended use is not permitted by statutory regulation or exceeds the permitted use, you will need to obtain permission directly from the copyright holder. To view a copy of this licence, visit http://creativecommons. org/licenses/by/4.0/.

\section{References}

1. Tsujimoto S, Genovesio A, Wise SP. Evaluating self-generated decisions in frontal pole cortex of monkeys. Nat Neurosci 2010, 13: $120-126$.

2. Passingham R. How good is the macaque monkey model of the human brain? Curr Opin Neurobiol 2009, 19: 6-11.

3. Gibbs RA, Rogers J, Katze MG, Bumgarner R, Weinstock GM, Mardis ER, et al. Evolutionary and biomedical insights from the rhesus macaque genome. Science 2007, 316: 222-234.

4. Gebhard R, Zilles K, Schleicher A, Everitt BJ, Robbins TW, Divac I. Parcellation of the frontal cortex of the New World monkey Callithrix jacchus by eight neurotransmitter-binding sites. Anat Embryol (Berl) 1995, 191: 509-517.

5. Fuster JnM. The prefrontal cortex-An update: Time is of the essence. Neuron 2001, 30: 319-333.

6. Rushworth MF, Noonan MP, Boorman ED, Walton ME, Behrens TE. Frontal cortex and reward-guided learning and decision-making. Neuron 2011, 70: 1054-1069.

7. Koechlin E, Hyafil A. Anterior prefrontal function and the limits of human decision-making. Science 2007, 318: 594-598.

8. Neubert FX, Mars RB, Thomas AG, Sallet J, Rushworth MF. Comparison of human ventral frontal cortex areas for cognitive control and language with areas in monkey frontal cortex. Neuron 2014, 81: 700-713.

9. Mansouri FA, Koechlin E, Rosa MGP, Buckley MJ. Managing competing goals - a key role for the frontopolar cortex. Nat Rev Neurosci 2017, 18: 645-657.
10. Tsujimoto S, Genovesio A, Wise SP. Frontal pole cortex: encoding ends at the end of the endbrain. Trends Cogn Sci 2011, 15: 169-176.

11. Boschin EA, Piekema C, Buckley MJ. Essential functions of primate frontopolar cortex in cognition. Proc Natl Acad Sci U S A 2015, 112: E1020-E1027.

12. Germuska M, Saha S, Fiala J, Barbas H. Synaptic distinction of laminar-specific prefrontal-temporal pathways in primates. Cereb Cortex 2006, 16: 865-875.

13. Joyce MKP, Barbas H. Cortical connections position primate prea 25 as a keystone for interoception, emotion, and memory. J Neurosci 2018, 38: 1677-1698.

14. Medalla M, Barbas H. Anterior cingulate synapses in prefrontal areas 10 and 46 suggest differential influence in cognitive control. J Neurosci 2010, 30: 16068-16081.

15. Medalla M, Lera P, Feinberg M, Barbas H. Specificity in inhibitory systems associated with prefrontal pathways to temporal cortex in primates. Cereb Cortex 2007, 17 Suppl 1: i136-i150.

16. Brodmann K. Vergleichende Lokalisationslehre der Grosshirnrinde in ihren Prinzipien dargestellt auf Grund des Zellenbaues. Barth, 1909.

17. Walker AE. A cytoarchitectural study of the prefrontal area of the macaque monkey. Journal of Comparative Neurology 1940, 73: $59-86$.

18. Paxinos G, Huang XF, Toga AW. The Rhesus Monkey Brain: in Stereotaxic Coordinates. (2 ed.). 2009.

19. Saleem KS, Kondo H, Price JL. Complementary circuits connecting the orbital and medial prefrontal networks with the temporal, insular, and opercular cortex in the macaque monkey. J Comp Neurol 2008, 506: 659-693.

20. Carmichael ST, Price JL. Architectonic subdivision of the orbital and medial prefrontal cortex in the macaque monkey. J Comp Neurol 1994, 346: 366-402.

21. Mantini D, Vanduffel W. Emerging roles of the brain's default network. Neuroscientist 2013, 19: 76-87.

22. Mantini D, Gerits A, Nelissen K, Durand JB, Joly O, Simone L, et al. Default mode of brain function in monkeys. J Neurosci 2011, 31: 12954-12962.

23. Sliwa J, Freiwald WA. A dedicated network for social interaction processing in the primate brain. Science 2017, 356: 745-749.

24. Miyamoto K, Setsuie R, Osada T, Miyashita Y. Reversible silencing of the frontopolar cortex selectively impairs metacognitive judgment on non-experience in primates. Neuron 2018, 97: 980-989 e986.

25. Mansouri FA, Buckley MJ, Mahboubi M, Tanaka K. Behavioral consequences of selective damage to frontal pole and posterior cingulate cortices. Proc Natl Acad Sci U S A 2015, 112: E3940E3949.

26. Saleem KS, Miller B, Price JL. Subdivisions and connectional networks of the lateral prefrontal cortex in the macaque monkey. J Comp Neurol 2014, 522: 1641-1690.

27. Barbas H, Pandya DN. Architecture and intrinsic connections of the prefrontal cortex in the rhesus monkey. J Comp Neurol 1989, 286: 353-375.

28. Fan L, Li H, Zhuo J, Zhang Y, Wang J, Chen L, et al. The human brainnetome atlas: A new brain atlas based on connectional architecture. Cereb Cortex 2016, 26: 3508-3526.

29. Eickhoff SB, Yeo BTT, Genon S. Imaging-based parcellations of the human brain. Nat Rev Neurosci 2018, 19: 672-686.

30. Cerliani L, D'Arceuil H, Thiebaut de Schotten M. Connectivitybased parcellation of the macaque frontal cortex, and its relation with the cytoarchitectonic distribution described in current atlases. Brain Struct Funct 2017, 222: 1331-1349. 
31. Assaf Y, Johansen-Berg H, Thiebaut de Schotten M. The role of diffusion MRI in neuroscience. NMR Biomed 2019, 32: e3762.

32. O'Donnell LJ, Daducci A, Wassermann D, Lenglet C. Advances in computational and statistical diffusion MRI. NMR Biomed 2019, 32: e3805.

33. Xia X, Fan L, Hou B, Zhang B, Zhang D, Cheng C, et al. Finegrained parcellation of the macaque nucleus accumbens by highresolution diffusion tensor tractography. Front Neurosci 2019, 13: 709 .

34. Calabrese E, Badea A, Coe CL, Lubach GR, Shi Y, Styner MA, et al. A diffusion tensor MRI atlas of the postmortem rhesus macaque brain. Neuroimage 2015, 117: 408-416.

35. Balbastre Y, Riviere D, Souedet N, Fischer C, Herard AS, Williams S, et al. A validation dataset for Macaque brain MRI segmentation. Data Brief 2018, 16: 37-42.

36. Haile TM, Bohon KS, Romero MC, Conway BR. Visual stimulus-driven functional organization of macaque prefrontal cortex. Neuroimage 2019, 188: 427-444.

37. Tasserie J, Grigis A, Uhrig L, Dupont M, Amadon A, Jarraya B. Pypreclin: An automatic pipeline for macaque functional MRI preprocessing. Neuroimage 2019: 116353.

38. Yushkevich PA, Piven J, Hazlett HC, Smith RG, Ho S, Gee JC, et al. User-guided 3D active contour segmentation of anatomical structures: significantly improved efficiency and reliability. Neuroimage 2006, 31: 1116-1128.

39. Xu J, Wang J, Fan L, Li H, Zhang W, Hu Q, et al. Tractographybased parcellation of the human middle temporal gyrus. Sci Rep 2015, 5: 18883.

40. Avants BB, Tustison NJ, Song G, Cook PA, Klein A, Gee JC. A reproducible evaluation of ANTs similarity metric performance in brain image registration. Neuroimage 2011, 54: 2033-2044.

41. Liang S, Wang Q, Kong X, Deng W, Yang X, Li X, et al. White matter abnormalities in major depression biotypes identified by diffusion tensor imaging. Neurosci Bull 2019, 35: 867-876.

42. He B, Yang Z, Fan L, Gao B, Li H, Ye C, et al. MonkeyCBP: A toolbox for connectivity-based parcellation of monkey brain. Front Neuroinform 2020, 14: 14.

43. Wang J, Zuo Z, Xie S, Miao Y, Ma Y, Zhao X, et al. Parcellation of macaque cortex with anatomical connectivity profiles. Brain Topogr 2018, 31: 161-173.

44. Behrens TE, Berg HJ, Jbabdi S, Rushworth MF, Woolrich MW. Probabilistic diffusion tractography with multiple fibre orientations: What can we gain? Neuroimage 2007, 34: 144-155.

45. Sani I, McPherson BC, Stemmann H, Pestilli F, Freiwald WA. Functionally defined white matter of the macaque monkey brain reveals a dorso-ventral attention network. Elife 2019, 8.

46. Takemura H, Caiafa CF, Wandell BA, Pestilli F. Ensemble Tractography. PLoS Comput Biol 2016, 12: e1004692.

47. Johansen-Berg H, Behrens TE, Robson MD, Drobnjak I, Rushworth MF, Brady JM, et al. Changes in connectivity profiles define functionally distinct regions in human medial frontal cortex. Proc Natl Acad Sci U S A 2004, 101: 13335-13340.

48. Zhuo J, Fan L, Liu Y, Zhang Y, Yu C, Jiang T. Connectivity profiles reveal a transition subarea in the parahippocampal region that integrates the anterior temporal-posterior medial systems. J Neurosci 2016, 36: 2782-2795.

49. Fan L, Wang J, Zhang Y, Han W, Yu C, Jiang T. Connectivitybased parcellation of the human temporal pole using diffusion tensor imaging. Cereb Cortex 2014, 24: 3365-3378.

50. Liu H, Qin W, Li W, Fan L, Wang J, Jiang T, et al. Connectivity-based parcellation of the human frontal pole with diffusion tensor imaging. J Neurosci 2013, 33: 6782-6790.

51. Tungaraza RL, Mehta SH, Haynor DR, Grabowski TJ. Anatomically informed metrics for connectivity-based cortical parcellation from diffusion MRI. IEEE J Biomed Health Inform 2015, 19: 1375-1383.

52. Bzdok D, Heeger A, Langner R, Laird AR, Fox PT, PalomeroGallagher N, et al. Subspecialization in the human posterior medial cortex. Neuroimage 2015, 106: 55-71.

53. Kelly C, Uddin LQ, Shehzad Z, Margulies DS, Castellanos FX, Milham MP, et al. Broca's region: linking human brain functional connectivity data and non-human primate tracing anatomy studies. Eur J Neurosci 2010, 32: 383-398.

54. Thiebaut de Schotten M, Urbanski M, Valabregue R, Bayle DJ, Volle E. Subdivision of the occipital lobes: an anatomical and functional MRI connectivity study. Cortex 2014, 56: 121-137.

55. Wold S. Cross-validatory estimation of the number of components in factor and principal components models. Technometrics 1978, 20: 397-405.

56. Kaiser HF. The application of electronic computers to factor analysis. Edu Psychol Measur 1960, 20: 141-151.

57. Bakker R, Tiesinga P, Kotter R. The scalable brain atlas: Instant web-based access to public brain atlases and related content. Neuroinformatics 2015, 13: 353-366.

58. Miyamoto K, Osada T, Setsuie R, Takeda M, Tamura K, Adachi $\mathrm{Y}$, et al. Causal neural network of metamemory for retrospection in primates. Science 2017, 355: 188-193.

59. Xia X, Fan L, Cheng C, Cheng L, Cao L, He B, et al. Mapping connectional differences between humans and macaques in the nucleus accumbens shell-core architecture. bioRxiv 2020: 2020.2006.2012.147546.

60. Suárez LE, Markello RD, Betzel RF, Misic B. Linking structure and function in macroscale brain networks. Trends Cogn Sci 2020, 24: 302-315

61. Chen Y, Wang S, Hilgetag CC, Zhou C. Features of spatial and functional segregation and integration of the primate connectome revealed by trade-off between wiring cost and efficiency. PLOS Computational Biology 2017, 13: e1005776.

62. Hagmann P, Cammoun L, Gigandet X, Meuli R, Honey CJ, Wedeen VJ, et al. Mapping the structural core of human cerebral cortex. PLoS Biol 2008, 6: e159.

63. Chen ZJ, He Y, Rosa-Neto P, Germann J, Evans AC. Revealing modular architecture of human brain structural networks by using cortical thickness from MRI. Cereb Cortex 2008, 18: 2374-2381.

64. Van Essen DC. Windows on the brain: the emerging role of atlases and databases in neuroscience. Curr Opin Neurobiol 2002, 12: 574-579.

65. Van Essen DC, Drury HA, Dickson J, Harwell J, Hanlon D, Anderson $\mathrm{CH}$. An integrated software suite for surface-based analyses of cerebral cortex. J Am Med Inform Assoc 2001, 8: 443-459.

66. Wu Z, Ainsworth M, Browncross H, Bell AH, Buckley MJ. Frontopolar cortex is a mediator of network modularity in the primate brain. bioRxiv 2019: 2019.2012.2020.882837.

67. Miranda-Dominguez O, Mills BD, Grayson D, Woodall A, Grant KA, Kroenke CD, et al. Bridging the gap between the human and macaque connectome: a quantitative comparison of global interspecies structure-function relationships and network topology. J Neurosci 2014, 34: 5552-5563.

68. Goulas A, Stiers P, Hutchison RM, Everling S, Petrides M, Margulies DS. Intrinsic functional architecture of the macaque dorsal and ventral lateral frontal cortex. J Neurophysiol 2017, 117: 1084-1099.

69. An X, Bandler R, Ongur D, Price JL. Prefrontal cortical projections to longitudinal columns in the midbrain periaqueductal gray in macaque monkeys. J Comp Neurol 1998, 401: 455-479.

70. Ongur D, An X, Price JL. Prefrontal cortical projections to the hypothalamus in macaque monkeys. J Comp Neurol 1998, 401: 480-505. 
71. Ongur D, Ferry AT, Price JL. Architectonic subdivision of the human orbital and medial prefrontal cortex. J Comp Neurol 2003, 460: 425-449.

72. Petrides M, Pandya DN. Efferent association pathways from the rostral prefrontal cortex in the macaque monkey. J Neurosci 2007, 27: 11573-11586.

73. Barbas H, Mesulam M. Cortical afferent input to the principals region of the rhesus monkey. Neuroscience 1985, 15: 619-637.

74. Barbas H, Ghashghaei H, Dombrowski SM, Rempel-Clower NL. Medial prefrontal cortices are unified by common connections with superior temporal cortices and distinguished by input from memory-related areas in the rhesus monkey. J Comp Neurol 1999, 410: 343-367.

75. Parvizi J, Van Hoesen GW, Buckwalter J, Damasio A. Neural connections of the posteromedial cortex in the macaque. Proc Natl Acad Sci U S A 2006, 103: 1563-1568.

76. Petrides M, Pandya DN. Dorsolateral prefrontal cortex: comparative cytoarchitectonic analysis in the human and the macaque brain and corticocortical connection patterns. Eur $\mathbf{J}$ Neurosci 1999, 11: 1011-1036.

77. Ferry AT, Ongur D, An X, Price JL. Prefrontal cortical projections to the striatum in macaque monkeys: Evidence for an organization related to prefrontal networks. J Comp Neurol 2000, 425: 447-470.

78. Ghashghaei HT, Hilgetag CC, Barbas H. Sequence of information processing for emotions based on the anatomic dialogue between prefrontal cortex and amygdala. Neuroimage 2007, 34 : 905-923.

79. Hsu DT, Price JL. Midline and intralaminar thalamic connections with the orbital and medial prefrontal networks in macaque monkeys. J Comp Neurol 2007, 504: 89-111.

80. Rempel-Clower NL, Barbas H. Topographic organization of connections between the hypothalamus and prefrontal cortex in the rhesus monkey. J Comp Neurol 1998, 398: 393-419.

81. Romanski LM, Giguere M, Bates JF, Goldman-Rakic PS. Topographic organization of medial pulvinar connections with the prefrontal cortex in the rhesus monkey. J Comp Neurol 1997, 379: 313-332.

82. Cho YT, Ernst M, Fudge JL. Cortico-amygdala-striatal circuits are organized as hierarchical subsystems through the primate amygdala. J Neurosci 2013, 33: 14017-14030.

83. Hilgetag CC, Burns GA, O’Neill MA, Scannell JW, Young MP. Anatomical connectivity defines the organization of clusters of cortical areas in the macaque monkey and the cat. Philos Trans R Soc Lond B Biol Sci 2000, 355: 91-110.

84. Carmichael ST, Price JL. Connectional networks within the orbital and medial prefrontal cortex of macaque monkeys. J Comp Neurol 1996, 371: 179-207.

85. Price JL, Carmichael ST, Drevets WC. Networks related to the orbital and medial prefrontal cortex; a substrate for emotional behavior? Prog Brain Res 1996, 107: 523-536.

86. Shepherd SV, Freiwald WA. Functional Networks for Social Communication in the Macaque Monkey. Neuron 2018, 99: 413-420 e413.

87. Mars RB, Passingham RE, Jbabdi S. Connectivity fingerprints: From areal descriptions to abstract spaces. Trends Cogn Sci 2018, 22: 1026-1037.

88. Jeurissen B, Descoteaux M, Mori S, Leemans A. Diffusion MRI fiber tractography of the brain. NMR Biomed 2019, 32: e3785.
89. Newcombe VF, Williams GB, Scoffings D, Cross J, Carpenter TA, Pickard JD, et al. Aetiological differences in neuroanatomy of the vegetative state: insights from diffusion tensor imaging and functional implications. J Neurol Neurosurg Psychiatry 2010, 81: 552-561.

90. Johansen-Berg H, Rushworth MF. Using diffusion imaging to study human connectional anatomy. Annu Rev Neurosci 2009, 32: 75-94.

91. Eickhoff SB, Thirion B, Varoquaux G, Bzdok D. Connectivitybased parcellation: Critique and implications. Hum Brain Mapp 2015, 36: 4771-4792.

92. Rilling JK, Glasser MF, Preuss TM, Ma X, Zhao T, Hu X, et al. The evolution of the arcuate fasciculus revealed with comparative DTI. Nat Neurosci 2008, 11: 426-428.

93. van den Heuvel MP, de Reus MA, Feldman Barrett L, Scholtens LH, Coopmans FM, Schmidt R, et al. Comparison of diffusion tractography and tract-tracing measures of connectivity strength in rhesus macaque connectome. Hum Brain Mapp 2015, 36: 3064-3075.

94. Azadbakht H, Parkes LM, Haroon HA, Augath M, Logothetis NK, de Crespigny A, et al. Validation of high-resolution tractography against in vivo tracing in the macaque visual cortex. Cereb Cortex 2015, 25: 4299-4309.

95. Girard G, Descoteaux M. Anatomical Tissue Probability Priors for Tractography. 2012.

96. Shen K, Goulas A, Grayson DS, Eusebio J, Gati JS, Menon RS, et al. Exploring the limits of network topology estimation using diffusion-based tractography and tracer studies in the macaque cortex. Neuroimage 2019, 191: 81-92.

97. Sotiropoulos SN. Processing of diffusion MR images of the brain: from crossing fibres to distributed tractography. 2010.

98. Tournier J, Calamante F, Connelly A. Effect of step size on probabilistic streamlines: implications for the interpretation of connectivity analysis. Proc Intl Soc Mag Reson Med 2011, 19: 2019.

99. Tournier JD, Calamante F, Connelly A. MRtrix: Diffusion tractography in crossing fiber regions. Int $\mathrm{J}$ Imaging Syst Technol 2012, 22: 53-66.

100. Stephan KE. The history of CoCoMac. Neuroimage 2013, 80: 46-52.

101. Markov NT, Ercsey-Ravasz MM, Ribeiro Gomes AR, Lamy C, Magrou L, Vezoli J, et al. A weighted and directed interareal connectivity matrix for macaque cerebral cortex. Cereb Cortex 2014, 24: 17-36.

102. Thomas C, Ye FQ, Irfanoglu MO, Modi P, Saleem KS, Leopold DA, et al. Anatomical accuracy of brain connections derived from diffusion MRI tractography is inherently limited. Proc Natl Acad Sci U S A 2014, 111: 16574-16579.

103. Gao Y, Choe AS, Stepniewska I, Li X, Avison MJ, Anderson AW. Validation of DTI tractography-based measures of primary motor area connectivity in the squirrel monkey brain. PLoS One 2013, 8: e75065.

104. Jiang T. Recent progress in basic and clinical research on disorders of consciousness. Neurosci Bull 2018, 34: 589-591.

105. Song M, Zhang Y, Cui Y, Yang Y, Jiang T. Brain network studies in chronic disorders of consciousness: Advances and perspectives. Neurosci Bull 2018, 34: 592-604. 